Tasks Study (Ugly, Beautiful, Negative, Positive)- MATLAB Anova Results

\begin{tabular}{|c|c|c|}
\hline Attribute & Parameter & $\begin{array}{l}\text { Column\# } \\
\text { in results }\end{array}$ \\
\hline \multirow{5}{*}{ Time } & $\%$ playing time & 2 \\
\hline & $\%$ idle time & 4 \\
\hline & $\%$ start time & 46 \\
\hline & $\%$ concurrent & 3 \\
\hline & total (minutes) & 1 \\
\hline \multirow{6}{*}{$\begin{array}{l}\text { Notes/ } \\
\text { Keys }\end{array}$} & \# of presses & 10 \\
\hline & $\%$ used & 11 \\
\hline & presses per key & 29 \\
\hline & play per key (sec) & 12 \\
\hline & \% black presses & 34 \\
\hline & $\%$ white presses & 35 \\
\hline \multirow{4}{*}{ Intensity $^{\dagger}$} & average & 9 \\
\hline & lowest (minimum) & 7 \\
\hline & highest (maximum) & 8 \\
\hline & most used & 5 \\
\hline \multirow{4}{*}{ Octave } & average & 21 \\
\hline & lowest (minimum) & 19 \\
\hline & highest (maximum) & 20 \\
\hline & most used & 17 \\
\hline \multirow{4}{*}{$\begin{array}{l}\text { Cluster } \\
\text { of notes }\end{array}$} & \# of instances & 24 \\
\hline & max pressed & 28 \\
\hline & most pressed $^{\S}$ & 25 \\
\hline & $\%$ most played $\|^{\|}$ & 26 \\
\hline \multirow{9}{*}{$\begin{array}{l}\text { Transit- } \\
\text { ions }\end{array}$} & \% diminuendo & 41 \\
\hline & $\%$ crescendo & 42 \\
\hline & $\%$ same intensity & 43 \\
\hline & $\%$ accelerando & 44 \\
\hline & $\%$ ritardando & 45 \\
\hline & $\%$ white to black & 37 \\
\hline & $\%$ black to white & 38 \\
\hline & $\%$ black to black & 39 \\
\hline & $\%$ white to white & 40 \\
\hline $\begin{array}{l}\text { Pitch } \\
\text { classes }\end{array}$ & $\begin{array}{l}\text { \% playing time: } \\
\text { C,C\#,D,D\#,E,F,F\#, } \\
\text { G,G\#,A,A\#,B }\end{array}$ & $48-59$ \\
\hline
\end{tabular}

\begin{tabular}{|c|c|}
\hline Variable & Definition \\
\hline \multicolumn{2}{|c|}{ Parameters matrix representation (pages 2-8) } \\
\hline 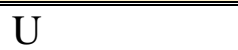 & "Ugly task mean \\
\hline USD & Ugly task SD \\
\hline USE & Ugly task SEM \\
\hline $\mathrm{B}$ & Beautiful task mean \\
\hline BSD & Beautiful task SD \\
\hline BSE & Beautiful task SEM \\
\hline $\mathrm{N}$ & Negative task mean \\
\hline NSD & Negative task SD \\
\hline NSE & Negative task SEM \\
\hline $\mathrm{P}$ & Positive task mean \\
\hline PSD & Positive task SD \\
\hline PSE & Positive task SEM \\
\hline PP & $\begin{array}{l}p \text {-value is the probability that the F- } \\
\text { statistic can take a value larger than } \\
\text { the computed test-statistic value. } \\
\text { Anova tests the null hypothesis that } \\
\text { all tasks means are equal to each other } \\
\text { against the alternative hypothesis that } \\
\text { at least one tasks mean is different } \\
\text { from the others. }\end{array}$ \\
\hline$\eta^{2}$ & Effect size \\
\hline \multicolumn{2}{|c|}{ Per parameter description (see page 9 onwards) } \\
\hline $\begin{array}{l}\mathrm{F} \text { (in anova } \\
\text { table) }\end{array}$ & $\begin{array}{l}\text { F-statistic, which is the ratio of the } \\
\text { mean squares. }\end{array}$ \\
\hline $\begin{array}{l}\text { df (in anova } \\
\text { table) }\end{array}$ & $\begin{array}{l}\text { The degrees of freedom associated } \\
\text { with each source. }\end{array}$ \\
\hline $\begin{array}{l}\text { multiple } \\
\text { comparison } \\
\text { (Bonferroni) } \\
\text { results table }\end{array}$ & $\begin{array}{l}\text { The first two columns show which } \\
\text { groups are compared. The fourth } \\
\text { column shows the mean parameter } \\
\text { difference for the compared groups. } \\
\text { The third and fifth columns show the } \\
\text { lower and upper limits for a 95\% } \\
\text { confidence interval for the difference } \\
\text { in the group means. The last column } \\
\text { shows the p-values for the tests. }\end{array}$ \\
\hline means & $\begin{array}{l}\text { mean estimates for each group of } \\
\text { tasks }\end{array}$ \\
\hline
\end{tabular}

https://www.mathworks.com/help/stats/anova1.html

https://www.mathworks.com/help/stats/multiple-comparisons.html

\footnotetext{
1-pppp ; 2-ppp ; 3-pp ; 4-p ; 5-mp ; 6-mf ; 7-f ; 8-ff ; 9-fff; 10-ffff (See Fig 4A)

‡ configuration of maximum number of keys pressed; ; ${ }^{\S}$ most pressed configuration ;

$\|$ relative playing time of the most pressed configuration;
} 
$\mathrm{U}=$

Columns 1 through 10

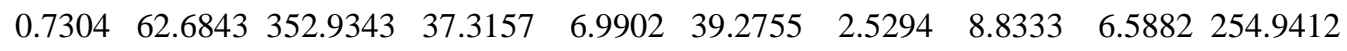

Columns 11 through 20

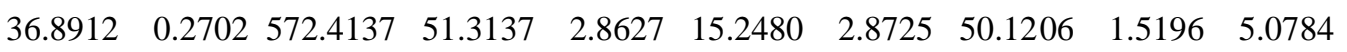

Columns 21 through 30

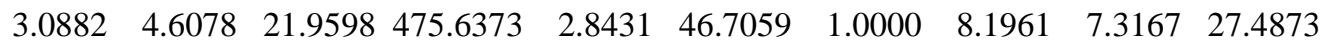

Columns 31 through 40

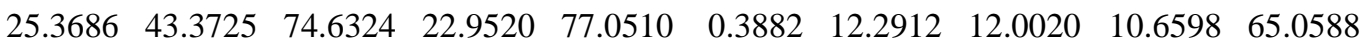

Columns 41 through 50

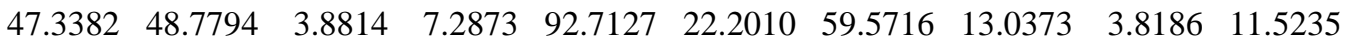

Columns 51 through 60

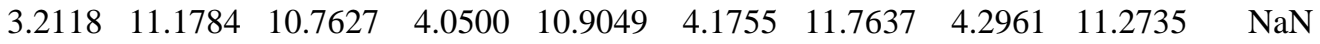

$\mathrm{B}=$

Columns 1 through 10

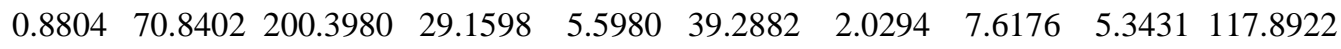

Columns 11 through 20

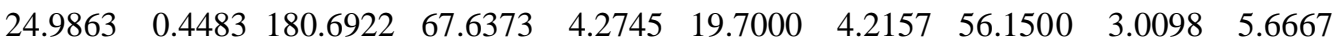

Columns 21 through 30

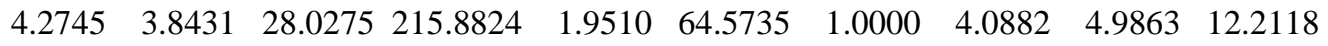

Columns 31 through 40

$\begin{array}{llllllllll}15.7324 & 33.7931 & 84.2686 & 13.1735 & 86.8265 & 0.1824 & 6.9196 & 6.6529 & 6.2549 & 80.1765\end{array}$

Columns 41 through 50

$\begin{array}{llllllllll}46.3961 & 50.0882 & 3.5127 & 12.9549 & 86.9990 & 16.8167 & 63.8471 & 17.0961 & 1.7588 & 13.1873\end{array}$

Columns 51 through 60

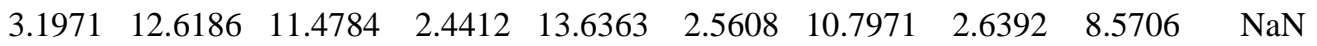


$\mathrm{N}=$

Columns 1 through 10

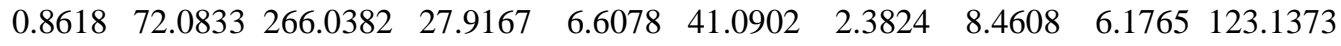

Columns 11 through 20

$\begin{array}{llllllllll}25.6882 & 0.5276 & 290.3980 & 48.2941 & 2.6275 & 20.8363 & 2.6569 & 58.9637 & 1.5098 & 4.5784\end{array}$

Columns 21 through 30

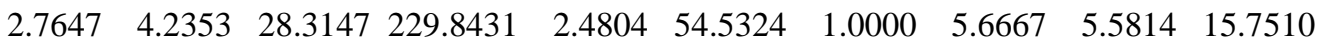

Columns 31 through 40

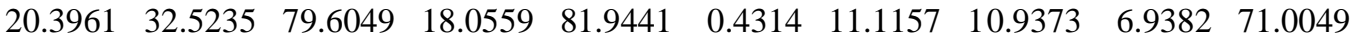

Columns 41 through 50

$\begin{array}{llllllllll}45.8422 & 50.3824 & 3.7784 & 13.3578 & 86.6235 & 16.4235 & 62.5529 & 14.9637 & 2.7892 & 10.0529\end{array}$

Columns 51 through 60

$\begin{array}{llllllllll}3.8088 & 13.3647 & 12.1245 & 3.9069 & 12.4392 & 2.9049 & 10.3059 & 3.4922 & 9.8529 & \mathrm{NaN}\end{array}$

$\mathrm{P}=$

Columns 1 through 10

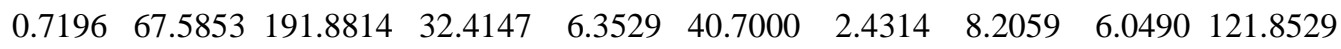

Columns 11 through 20

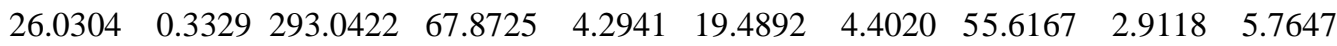

Columns 21 through 30

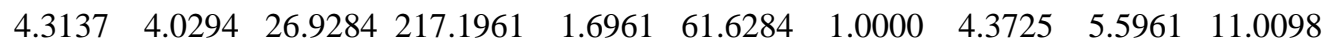

Columns 31 through 40

$\begin{array}{llllllllll}12.4108 & 36.3814 & 87.5931 & 8.8196 & 91.1804 & 0.1108 & 4.9598 & 4.9461 & 3.8608 & 86.2373\end{array}$

Columns 41 through 50

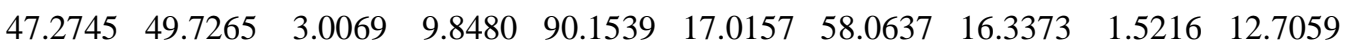

Columns 51 through 60

$\begin{array}{llllllllll}1.3853 & 13.4324 & 12.0735 & 1.8471 & 15.2304 & 1.4765 & 12.4382 & 1.4461 & 10.1029 & \mathrm{NaN}\end{array}$

$>$ 
$\mathrm{PP}=$

Columns 1 through 10

$\begin{array}{llllllllll}0.6335 & 0.0081 & 0.0000 & 0.0081 & 0.0000 & 0.6067 & 0.1013 & 0.0000 & 0.0000 & 0.0431\end{array}$

Columns 11 through 20

$\begin{array}{llllllllll}0.0000 & 0.0000 & 0.0000 & 0.0000 & 0.0000 & 0.0028 & 0.0000 & 0.0062 & 0.0000 & 0.0000\end{array}$

Columns 21 through 30

$\begin{array}{llllllllll}0.0000 & 0.3700 & 0.0001 & 0.0462 & 0.0000 & 0.0000 & \mathrm{NaN} & 0.0000 & 0.3040 & 0.0000\end{array}$

Columns 31 through 40

$\begin{array}{llllllllll}0.0000 & 0.0012 & 0.0000 & 0.0000 & 0.0000 & 0.0063 & 0.0000 & 0.0000 & 0.0027 & 0.0000\end{array}$

Columns 41 through 50

$\begin{array}{llllllllll}0.1987 & 0.2788 & 0.2479 & 0.0000 & 0.0000 & 0.0407 & 0.5083 & 0.1140 & 0.0007 & 0.0380\end{array}$

Columns 51 through 59

$\begin{array}{lllllllll}0.0189 & 0.2222 & 0.7075 & 0.0050 & 0.0042 & 0.0018 & 0.2571 & 0.0008 & 0.1283\end{array}$

Eta-sqaure $=$

Columns 1 through 10

$\left[\begin{array}{lllllllllll}{[0.0042]} & {[0.0287]} & {[0.1268]} & {[0.0287]} & {[0.0985]} & {[0.0045]} & {[0.0153]} & {[0.1217]} & {[0.1132]} & {[0.0199]}\end{array}\right.$

Columns 11 through 20

$\left[\begin{array}{lllllllllll}0.0681] & {[0.0730]} & {[0.0865]} & {[0.2784]} & {[0.2837]} & {[0.0342]} & {[0.3094]} & {[0.0302]} & {[0.2978]} & {[0.1168]}\end{array}\right.$

Columns 21 through 30

$\left[\begin{array}{llllllllllll}{[0.3388]} & {[0.0077]} & {[0.0489]} & {[0.0196]} & {[0.0572]} & {[0.0725]} & {[\mathrm{NaN}]} & {[0.1637]} & {[0.0089]} & {[0.0903]}\end{array}\right.$

Columns 31 through 40

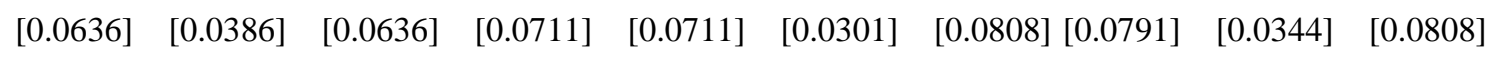

Columns 41 through 30

$\left[\begin{array}{lllllllllll}{[0.0114]} & {[0.0095]} & {[0.0102]} & {[0.0583]} & {[0.0586]} & {[0.0202]} & {[0.0057]} & {[0.0146]} & {[0.0411]} & {[0.0206]}\end{array}\right.$

Columns 51 through 59

$\left[\begin{array}{lllllllllll}{[0.0243]} & {[0.0108]} & {[0.0034]} & {[0.0312]} & {[0.0321]} & {[0.0366]} & {[0.0099]} & {[0.0408]} & {[0.0139]}\end{array}\right.$ 
$\mathrm{USD}=$

$1.0 \mathrm{e}+03 *$

Columns 1 through 10

$\begin{array}{llllllllll}0.0010 & 0.0201 & 0.2439 & 0.0201 & 0.0016 & 0.0119 & 0.0018 & 0.0012 & 0.0013 & 0.7398\end{array}$

Columns 11 through 20

$\begin{array}{llllllllll}0.0232 & 0.0002 & 0.8062 & 0.0156 & 0.0014 & 0.0102 & 0.0012 & 0.0167 & 0.0009 & 0.0015\end{array}$

Columns 21 through 30

$\begin{array}{llllllllll}0.0009 & 0.0032 & 0.0098 & 1.4195 & 0.0022 & 0.0239 & 0 & 0.0057 & 0.0158 & 0.0265\end{array}$

Columns 31 through 40

$\begin{array}{llllllllll}0.0180 & 0.0243 & 0.0180 & 0.0190 & 0.0190 & 0.0004 & 0.0110 & 0.0109 & 0.0106 & 0.0290\end{array}$

Columns 41 through 50

$\begin{array}{llllllllll}0.0057 & 0.0058 & 0.0036 & 0.0080 & 0.0080 & 0.0179 & 0.0290 & 0.0095 & 0.0042 & 0.0078\end{array}$

Columns 51 through 60

$\begin{array}{llllllllll}0.0039 & 0.0078 & 0.0081 & 0.0044 & 0.0068 & 0.0054 & 0.0074 & 0.0056 & 0.0075 & \mathrm{NaN}\end{array}$

USE $=$

Columns 1 through 10

$\begin{array}{llllllllll}0.0974 & 1.9938 & 24.1496 & 1.9938 & 0.1548 & 1.1807 & 0.1761 & 0.1175 & 0.1280 & 73.2536\end{array}$

Columns 11 through 20

$\begin{array}{llllllllll}2.2981 & 0.0217 & 79.8264 & 1.5413 & 0.1365 & 1.0057 & 0.1228 & 1.6531 & 0.0844 & 0.1505\end{array}$

Columns 21 through 30

$\begin{array}{llllllllll}0.0860 & 0.3178 & 0.9745 & 140.5472 & 0.2189 & 2.3622 & 0 & 0.5620 & 1.5684 & 2.6230\end{array}$

Columns 31 through 40

$\begin{array}{llllllllll}1.7784 & 2.4032 & 1.7785 & 1.8818 & 1.8817 & 0.0384 & 1.0908 & 1.0762 & 1.0519 & 2.8709\end{array}$

Columns 41 through 50

$\begin{array}{llllllllll}0.5655 & 0.5736 & 0.3591 & 0.7873 & 0.7873 & 1.7715 & 2.8678 & 0.9373 & 0.4132 & 0.7707\end{array}$

Columns 51 through 60

$\begin{array}{llllllllll}0.3832 & 0.7694 & 0.7987 & 0.4333 & 0.6744 & 0.5362 & 0.7310 & 0.5580 & 0.7461 & \mathrm{NaN}\end{array}$ 
$\mathrm{BSD}=$

Columns 1 through 10

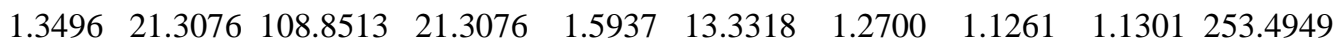

Columns 11 through 20

$\begin{array}{llllllllll}15.9158 & 0.2396 & 109.7490 & 14.5110 & 1.2118 & 11.8091 & 1.1400 & 18.3101 & 1.4316 & 1.0082\end{array}$

Columns 21 through 30

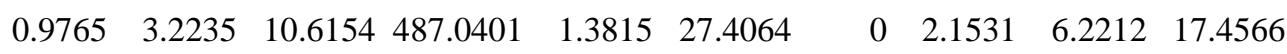

Columns 31 through 40

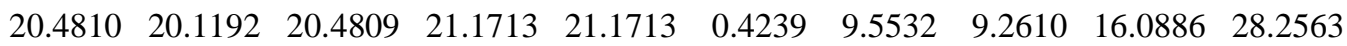

Columns 41 through 50

$\begin{array}{llllllllll}6.0140 & 5.7744 & 3.1981 & 10.8862 & 10.9844 & 15.8787 & 31.2393 & 14.2146 & 4.2041 & 7.8457\end{array}$

Columns 51 through 60

$\begin{array}{llllllllll}7.5465 & 7.1746 & 8.0676 & 6.0930 & 9.0528 & 5.8873 & 8.6613 & 5.2641 & 6.9623 & \mathrm{NaN}\end{array}$

$\mathrm{BSE}=$

Columns 1 through 10

$\begin{array}{llllllllll}0.1336 & 2.1098 & 10.7779 & 2.1098 & 0.1578 & 1.3200 & 0.1258 & 0.1115 & 0.1119 & 25.0997\end{array}$

Columns 11 through 20

$\begin{array}{llllllllll}1.5759 & 0.0237 & 10.8668 & 1.4368 & 0.1200 & 1.1693 & 0.1129 & 1.8130 & 0.1417 & 0.0998\end{array}$

Columns 21 through 30

$\begin{array}{llllllllll}0.0967 & 0.3192 & 1.0511 & 48.2242 & 0.1368 & 2.7136 & 0 & 0.2132 & 0.6160 & 1.7285\end{array}$

Columns 31 through 40

$\begin{array}{llllllllll}2.0279 & 1.9921 & 2.0279 & 2.0963 & 2.0963 & 0.0420 & 0.9459 & 0.9170 & 1.5930 & 2.7978\end{array}$

Columns 41 through 50

$\begin{array}{llllllllll}0.5955 & 0.5718 & 0.3167 & 1.0779 & 1.0876 & 1.5722 & 3.0931 & 1.4075 & 0.4163 & 0.7768\end{array}$

Columns 51 through 60

$\begin{array}{llllllllll}0.7472 & 0.7104 & 0.7988 & 0.6033 & 0.8964 & 0.5829 & 0.8576 & 0.5212 & 0.6894 & \mathrm{NaN}\end{array}$ 
$\mathrm{NSD}=$

Columns 1 through 10

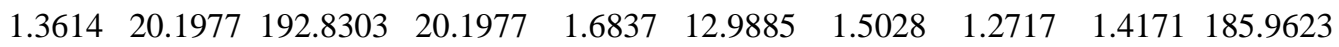

Columns 11 through 20

$\begin{array}{llllllllll}18.4790 & 0.5991 & 419.8788 & 14.6467 & 1.2342 & 13.1345 & 1.2308 & 19.7170 & 0.8052 & 1.6735\end{array}$

Columns 21 through 30

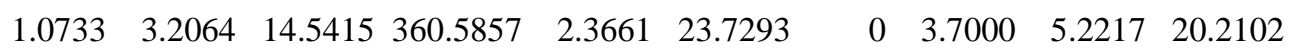

Columns 31 through 40

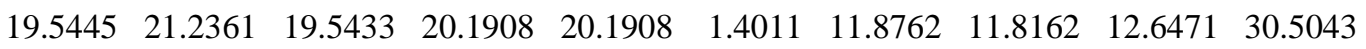

Columns 41 through 50

$\begin{array}{llllllllll}6.9754 & 7.8332 & 3.7417 & 10.5389 & 10.5566 & 15.9902 & 30.8011 & 15.5558 & 4.9976 & 9.0590\end{array}$

Columns 51 through 60

$\begin{array}{llllllllll}7.1720 & 11.8647 & 10.8870 & 6.3714 & 8.6052 & 4.8159 & 9.3309 & 5.8439 & 8.4108 & \mathrm{NaN}\end{array}$

$\mathrm{NSE}=$

Columns 1 through 10

$\begin{array}{llllllllll}0.1348 & 1.9999 & 19.0930 & 1.9999 & 0.1667 & 1.2860 & 0.1488 & 0.1259 & 0.1403 & 18.4130\end{array}$

Columns 11 through 20

$\begin{array}{llllllllll}1.8297 & 0.0593 & 41.5742 & 1.4502 & 0.1222 & 1.3005 & 0.1219 & 1.9523 & 0.0797 & 0.1657\end{array}$

Columns 21 through 30

$\begin{array}{llllllllll}0.1063 & 0.3175 & 1.4398 & 35.7033 & 0.2343 & 2.3496 & 0 & 0.3664 & 0.5170 & 2.0011\end{array}$

Columns 31 through 40

$\begin{array}{llllllllll}1.9352 & 2.1027 & 1.9351 & 1.9992 & 1.9992 & 0.1387 & 1.1759 & 1.1700 & 1.2523 & 3.0204\end{array}$

Columns 41 through 50

$\begin{array}{llllllllll}0.6907 & 0.7756 & 0.3705 & 1.0435 & 1.0453 & 1.5833 & 3.0498 & 1.5403 & 0.4948 & 0.8970\end{array}$

Columns 51 through 60

$\begin{array}{llllllllll}0.7101 & 1.1748 & 1.0780 & 0.6309 & 0.8520 & 0.4768 & 0.9239 & 0.5786 & 0.8328 & \mathrm{NaN}\end{array}$ 
$\mathrm{PSD}=$

Columns 1 through 10

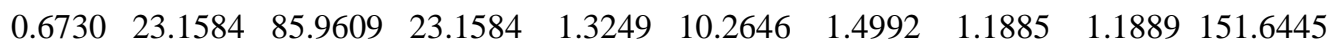

Columns 11 through 20

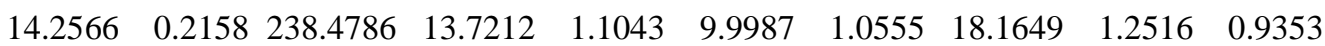

Columns 21 through 30

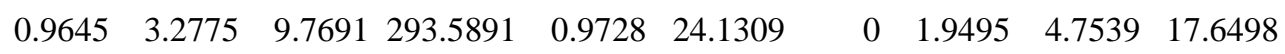

Columns 31 through 40

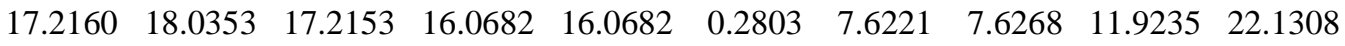

Columns 41 through 50

$\begin{array}{llllllllll}4.3629 & 5.0804 & 2.7802 & 10.2795 & 10.2802 & 16.4214 & 30.8482 & 10.7326 & 4.2671 & 8.7586\end{array}$

Columns 51 through 60

$\begin{array}{llllllllll}3.2494 & 7.1936 & 10.3573 & 3.7987 & 10.1922 & 3.3924 & 7.7414 & 3.5094 & 9.3473 & \mathrm{NaN}\end{array}$

PSE $=$

Columns 1 through 10

$\begin{array}{llllllllll}0.0666 & 2.2930 & 8.5114 & 2.2930 & 0.1312 & 1.0163 & 0.1484 & 0.1177 & 0.1177 & 15.0150\end{array}$

Columns 11 through 20

$\begin{array}{llllllllll}1.4116 & 0.0214 & 23.6129 & 1.3586 & 0.1093 & 0.9900 & 0.1045 & 1.7986 & 0.1239 & 0.0926\end{array}$

Columns 21 through 30

$\begin{array}{llllllllll}0.0955 & 0.3245 & 0.9673 & 29.0697 & 0.0963 & 2.3893 & 0 & 0.1930 & 0.4707 & 1.7476\end{array}$

Columns 31 through 40

$\begin{array}{llllllllll}1.7046 & 1.7858 & 1.7046 & 1.5910 & 1.5910 & 0.0278 & 0.7547 & 0.7552 & 1.1806 & 2.1913\end{array}$

Columns 41 through 50

$\begin{array}{llllllllll}0.4320 & 0.5030 & 0.2753 & 1.0178 & 1.0179 & 1.6260 & 3.0544 & 1.0627 & 0.4225 & 0.8672\end{array}$

Columns 51 through 60

$\begin{array}{llllllllll}0.3217 & 0.7123 & 1.0255 & 0.3761 & 1.0092 & 0.3359 & 0.7665 & 0.3475 & 0.9255 & \mathrm{NaN}\end{array}$

$>$ 


\section{Explanation of output format}

(See https://www.mathworks.com/help/stats/anova1.html; https://www.mathworks.com/help/stats/multiple-comparisons.html)

\begin{tabular}{|l|l|}
\hline \hline Anova Table & Definition \\
\hline \hline source & The source of the variability \\
\hline SS & The sum of squares due to each source \\
\hline df & The degrees of freedom associated with each source. \\
\hline MS & The mean squares for each source; SS/df \\
\hline F & F-statistic, which is the ratio of the mean squares. \\
\hline Prob $>\mathrm{F}$ & $\begin{array}{l}\text { The } p \text { value probability that the } \mathrm{F} \text { statisticcan take a } \\
\text { value larget than the computed test-statistic value. }\end{array}$ \\
\hline Columns & Variability between groups \\
\hline Error & Variability within groups \\
\hline Total & Total variability \\
\hline
\end{tabular}

$\begin{array}{lccccc}\text { 'Source' } & \text { 'SS' } & \text { 'df' } & \text { 'MS' } & \text { 'F' } & \text { 'Prob>F' } \\ \text { 'Columns' } & {[1.1409 \mathrm{e}+04]} & {[\mathrm{3}]} & {[3.8030 \mathrm{e}+03]} & {[10.3114]} & {[1.4886 \mathrm{e}-06]} \\ \text { 'Error' } & {[1.4900 \mathrm{e}+05]} & {[404]} & {[368.8187]} & {[]} & {[]} \\ \text { 'Total' } & {[1.6041 \mathrm{e}+05]} & {[407]} & {[]} & {[]} & {[]}\end{array}$

$\begin{array}{llcccc}1.0000 & 2.0000 & 2.6485 & 9.7784 & 16.9083 & 0.0019 \\ 1.0000 & 3.0000 & -2.2338 & 4.8961 & 12.0260 & 0.4164 \\ 1.0000 & 4.0000 & 7.0025 & 14.1324 & 21.2622 & 0.0000 \\ 2.0000 & 3.0000 & -12.0122 & -4.8824 & 2.2475 & 0.4211 \\ 2.0000 & 4.0000 & -2.7760 & 4.3539 & 11.4838 & 0.6373 \\ 3.0000 & 4.0000 & 2.1064 & 9.2363 & 16.3662 & 0.0039\end{array}$

Multiple comparison (Bonferroni) results table

The first two columns show which groups are compared. The fourth column shows the mean parameter difference for the compared groups. The third and fifth columns show the lower and upper limits for a $95 \%$ confidence interval for the difference in the group means. The last column shows the $\mathrm{p}$-values for the tests

\begin{tabular}{|c|c|c|}
\hline 22.9520 & 1.9015 & Means table \\
\hline 13.1735 & 1.9015 & mean estimates for each group of tasks, and \\
\hline 3.05 & $\begin{array}{c}1.9015 \\
1.9015\end{array}$ & standard errors of the estimates \\
\hline
\end{tabular}




\begin{tabular}{|c|c|c|c|c|c|}
\hline \multirow{2}{*}{$\begin{array}{l}\text { 'Source' } \\
\text { 'Columns' }\end{array}$} & & \multicolumn{2}{|c|}{ 'df' 'MS' } & 'F' & \\
\hline & $\mathrm{s}^{\prime} \quad$ [ 2.1 & 867] [ & 3] $[0.72$ & 89] & $0.5723]$ \\
\hline 'Error' & {$[514.58$} & 21] & {$[1.27$} & 37] & [] \\
\hline 'Total' & {$[516.76$} & $88]$ & 7] & [] & [] \\
\hline 1.0000 & 2.0000 & -0.5680 & -0.1490 & 0.2700 & 1.0000 \\
\hline 1.0000 & 3.0000 & -0.5494 & -0.1304 & 0.2886 & 1.0000 \\
\hline 1.0000 & 4.0000 & -0.4072 & 0.0118 & 0.4308 & 1.0000 \\
\hline 2.0000 & 3.0000 & -0.4004 & 0.0186 & 0.4376 & 1.0000 \\
\hline 2.0000 & 4.0000 & -0.2582 & 0.1608 & 0.5798 & 1.0000 \\
\hline 3.0000 & 4.0000 & -0.2768 & 0.1422 & 0.5612 & 1.0000 \\
\hline 0.7314 & 0.1117 & & & & \\
\hline 0.8804 & 0.1117 & & & & \\
\hline 0.8618 & 0.1117 & & & & \\
\hline 0.7196 & 0.1117 & & & & \\
\hline
\end{tabular}

2

'Source' 'SS' 'df' 'MS' 'F' 'Prob>F'

$\begin{array}{lllllll}\text { 'Columns' [5.3869e+03] [ 3] } & {[1.7956 \mathrm{e}+03]} & \text { [3.9820] } & \text { [0.0081] }\end{array}$

'Error' [1.8218e+05] [404] [ 450.9357] [] []

'Total' [1.8756e+05] [407] [] [] []

$\begin{array}{llllll}1.0000 & 2.0000 & -16.0396 & -8.1559 & -0.2721 & 0.0382\end{array}$

$\begin{array}{llllll}1.0000 & 3.0000 & -17.2828 & -9.3990 & -1.5153 & 0.0101\end{array}$

$\begin{array}{llllll}1.0000 & 4.0000 & -12.7847 & -4.9010 & 2.9828 & 0.6005\end{array}$

$\begin{array}{llllll}2.0000 & 3.0000 & -9.1269 & -1.2431 & 6.6406 & 1.0000\end{array}$

$\begin{array}{llllll}2.0000 & 4.0000 & -4.6289 & 3.2549 & 11.1387 & 1.0000\end{array}$

$\begin{array}{llllll}3.0000 & 4.0000 & -3.3857 & 4.4980 & 12.3818 & 0.7868\end{array}$

$62.6843 \quad 2.1026$

$70.8402 \quad 2.1026$

$72.0833 \quad 2.1026$

$67.5853 \quad 2.1026$

3

\begin{tabular}{|c|c|c|c|c|}
\hline 'Source' & 'SS' & 'MS' & 'Prob $>F^{\prime}$ & \\
\hline 'Columns & $s^{\prime} \quad[1.6992 \mathrm{e}+06]$ & {$[5.6641 \mathrm{e}+05]$} & [19.5469] & [7.4884e-12] \\
\hline 'Error' & {$[1.1707 \mathrm{e}+07]$} & {$[2.8977 \mathrm{e}+04]$} & [] & [] \\
\hline 'Total' & {$[1.3406 \mathrm{e}+07]$} & [407] & {[]} & \\
\hline 1.0000 & $2.0000 \quad 89.3383$ & 152.5363215 .7343 & 0.0000 & \\
\hline 1.0000 & $3.0000 \quad 23.6981$ & 86.8961150 .0941 & 0.0018 & \\
\hline 1.0000 & $4.0000 \quad 97.8549$ & 161.0529224 .2509 & 0.0000 & \\
\hline 2.0000 & $3.0000-128.8382$ & $2-65.6402-2.4422$ & 0.0369 & \\
\hline 2.0000 & $4.0000-54.6813$ & $8.5167 \quad 71.7147$ & 1.0000 & \\
\hline 3.0000 & $4.0000 \quad 10.9589$ & $74.1569 \quad 137.3549$ & 0.0120 & \\
\hline 352.9343 & 16.8549 & & & \\
\hline 200.3980 & 16.8549 & & & \\
\hline 266.0382 & 16.8549 & & & \\
\hline 191.8814 & 16.8549 & & & \\
\hline
\end{tabular}




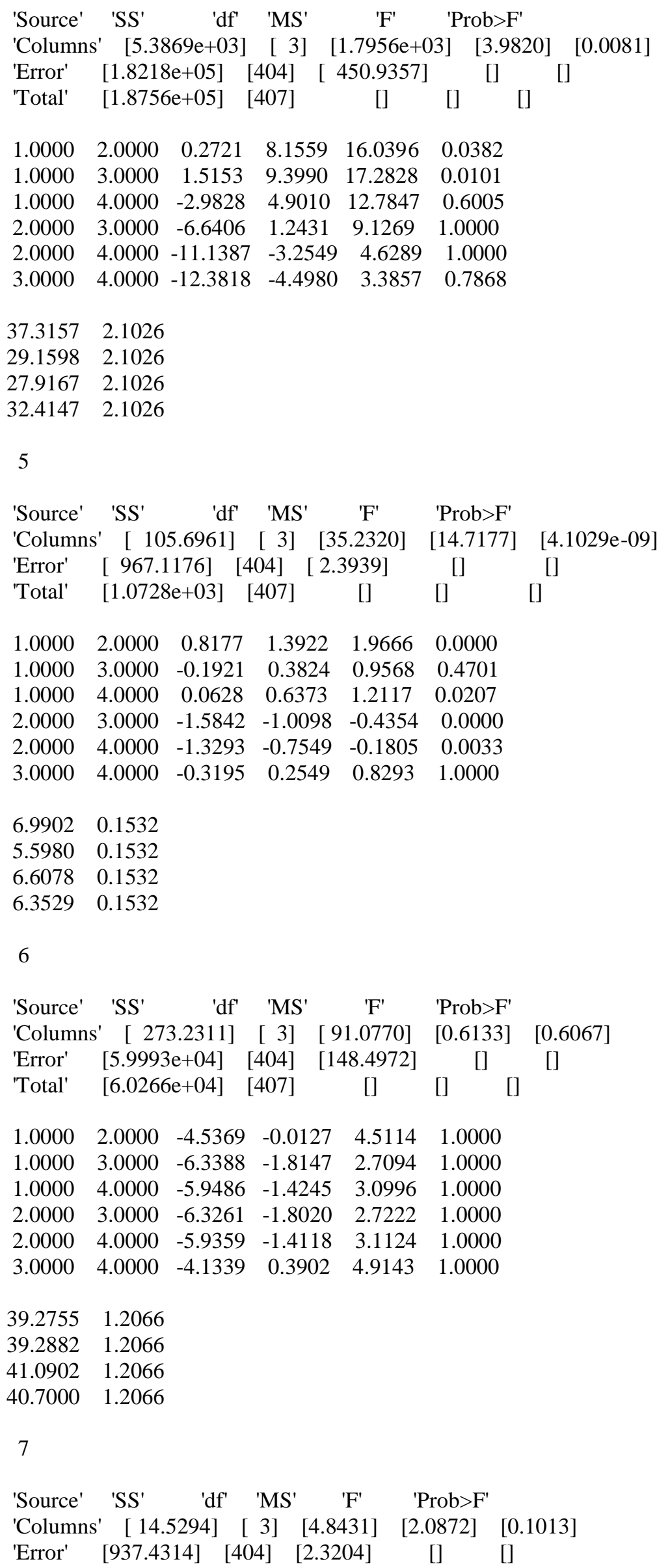




$\begin{array}{ccccccc}\text { 'Total' } & {[951.9608]} & {[407]} & {[]} & {[]} & {[]} \\ & & & & & \\ 1.0000 & 2.0000 & -0.0655 & 0.5000 & 1.0655 & 0.1173 \\ 1.0000 & 3.0000 & -0.4185 & 0.1471 & 0.7126 & 1.0000 \\ 1.0000 & 4.0000 & -0.4675 & 0.0980 & 0.6636 & 1.0000 \\ 2.0000 & 3.0000 & -0.9185 & -0.3529 & 0.2126 & 0.5926 \\ 2.0000 & 4.0000 & -0.9675 & -0.4020 & 0.1636 & 0.3613 \\ 3.0000 & 4.0000 & -0.6145 & -0.0490 & 0.5165 & 1.0000 \\ & & & & & \\ 2.5294 & 0.1508 & & & & \\ 2.0294 & 0.1508 & & & & \\ 2.3824 & 0.1508 & & & & \\ 2.4314 & 0.1508 & & & & \end{array}$

8

'Source' 'SS' 'df' 'MS' 'F' 'Prob>F'

'Columns' [ 79.8725] [ 3] [26.6242] [18.6650] [2.3391e-11]

$\begin{array}{llllll}\text { 'Error' [576.2745] [404] [ 1.4264] [] } & \text { [ ] }\end{array}$

'Total' [656.1471] [407] [] [] []

$\begin{array}{llllll}1.0000 & 2.0000 & 0.7723 & 1.2157 & 1.6591 & 0.0000\end{array}$

$\begin{array}{llllll}1.0000 & 3.0000 & -0.0709 & 0.3725 & 0.8160 & 0.1587\end{array}$

$\begin{array}{llllll}1.0000 & 4.0000 & 0.1840 & 0.6275 & 1.0709 & 0.0012\end{array}$

$\begin{array}{llllll}2.0000 & 3.0000 & -1.2865 & -0.8431 & -0.3997 & 0.0000\end{array}$

$\begin{array}{llllll}2.0000 & 4.0000 & -1.0316 & -0.5882 & -0.1448 & 0.0029\end{array}$

$\begin{array}{llllll}3.0000 & 4.0000 & -0.1885 & 0.2549 & 0.6983 & 0.7695\end{array}$

$8.8333 \quad 0.1183$

$7.6176 \quad 0.1183$

$8.4608 \quad 0.1183$

8.20590 .1183

9

'Source' 'SS' 'df' 'MS' 'F' 'Prob>F'

'Columns' [ 82.0980] [ 3] [27.3660] [17.1869] [1.5984e-10]

'Error' [643.2745] [404] [ 1.5923]

$\begin{array}{llllll}\text { 'Total' [725.3725] [407] [ ] } & \text { [] }\end{array}$

$\begin{array}{llllll}1.0000 & 2.0000 & 0.7766 & 1.2451 & 1.7136 & 0.0000\end{array}$

$\begin{array}{lllllll}1.0000 & 3.0000 & -0.0567 & 0.4118 & 0.8802 & 0.1217\end{array}$

$\begin{array}{lllllll}1.0000 & 4.0000 & 0.0707 & 0.5392 & 1.0077 & 0.0146\end{array}$

$\begin{array}{lllllll}2.0000 & 3.0000 & -1.3018 & -0.8333 & -0.3649 & 0.0000\end{array}$

$\begin{array}{lllllll}2.0000 & 4.0000 & -1.1744 & -0.7059 & -0.2374 & 0.0005\end{array}$

$\begin{array}{llllll}3.0000 & 4.0000 & -0.3410 & 0.1275 & 0.5959 & 1.0000\end{array}$

$\begin{array}{ll}6.5882 & 0.1249\end{array}$

$5.3431 \quad 0.1249$

6.17650 .1249

$6.0490 \quad 0.1249$

10

'Source' 'SS' 'df' 'MS' 'F' 'Prob>F'

$\begin{array}{llllll} & \text { 'Columns' [1.3748e+06] [ 3] [4.5825e+05] [2.7392] } & \text { [0.0431] }\end{array}$

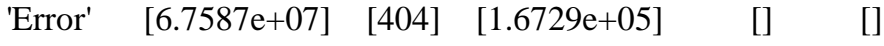

'Total' [6.8962e+07] [407] [] [] []

$\begin{array}{llllll}1.0000 & 2.0000 & -14.8018 & 137.0490 & 288.8998 & 0.1030\end{array}$ 


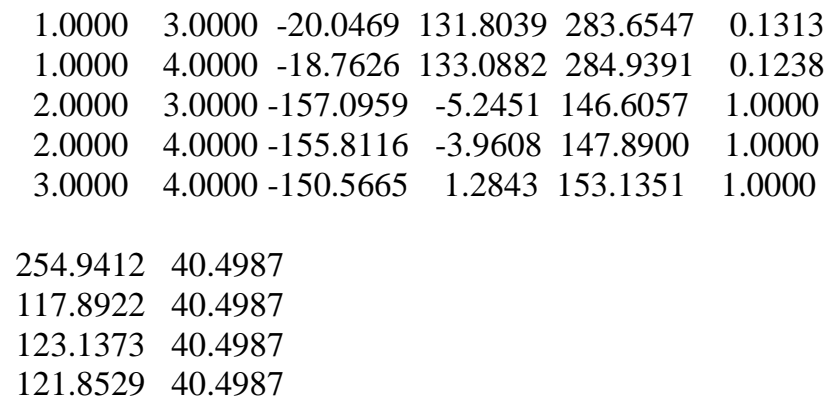

11

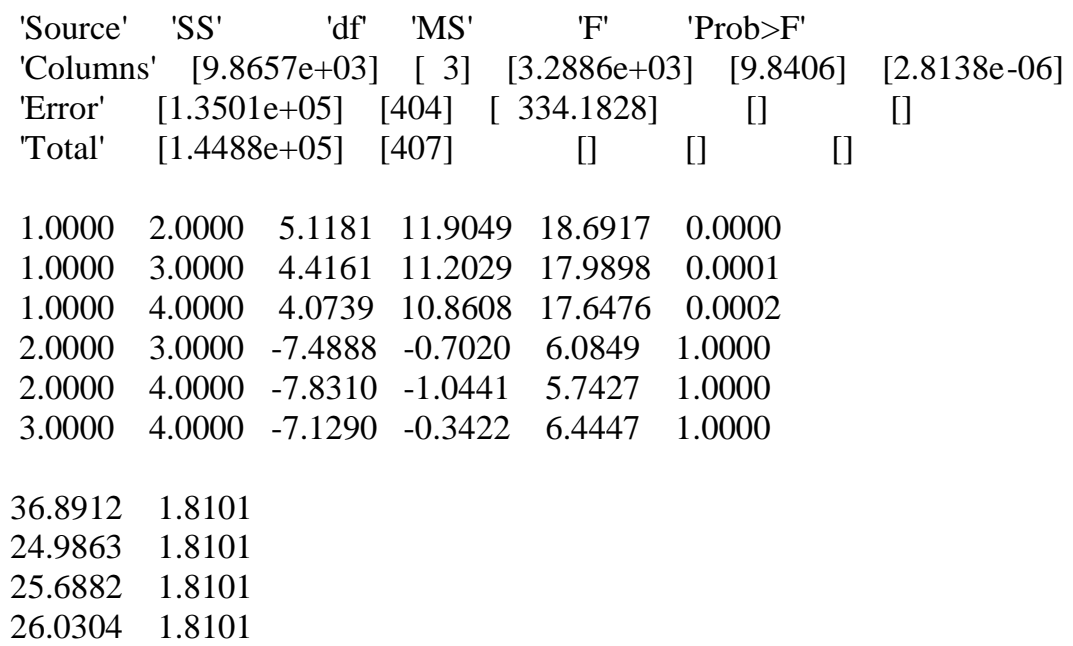

12

'Source' 'SS' 'df' 'MS' 'F'

'Columns' [ 4.0638] [ 3] [1.3546] [10.6024] [1.0049e-06]

$\begin{array}{llllll}\text { 'Error' [51.6168] [404] [0.1278] [] } & \text { [ }\end{array}$

'Total' [55.6806] [407] [] []

$\begin{array}{llllll}1.0000 & 2.0000 & -0.3107 & -0.1780 & -0.0453 & 0.0025\end{array}$

$\begin{array}{llllll}1.0000 & 3.0000 & -0.3901 & -0.2574 & -0.1246 & 0.0000\end{array}$

$\begin{array}{llllll}1.0000 & 4.0000 & -0.1953 & -0.0626 & 0.0701 & 1.0000\end{array}$

$\begin{array}{llllll}2.0000 & 3.0000 & -0.2120 & -0.0793 & 0.0534 & 0.6825\end{array}$

$\begin{array}{llllll}2.0000 & 4.0000 & -0.0173 & 0.1154 & 0.2481 & 0.1299\end{array}$

$\begin{array}{llllll}3.0000 & 4.0000 & 0.0620 & 0.1947 & 0.3274 & 0.0007\end{array}$

$0.2702 \quad 0.0354$

$0.4483 \quad 0.0354$

$0.5276 \quad 0.0354$

$0.3329 \quad 0.0354$

13

'Source' 'SS' 'df' 'MS' 'F' 'Prob>F'

'Columns' [8.5601e+06] [ 3] [2.8534e+06] [12.7499] [5.6168e-08]

$\begin{array}{llllll}\text { 'Error' [9.0414e+07] [404] [2.2380e+05] [] } & \text { [] }\end{array}$

'Total' [9.8974e+07] [407] [] [] []

$\begin{array}{llllll}1.0000 & 2.0000 & 216.0901 & 391.7216 & 567.3531 & 0.0000\end{array}$

$\begin{array}{lllllll}1.0000 & 3.0000 & 106.3842 & 282.0157 & 457.6472 & 0.0002\end{array}$

$\begin{array}{lllllll}1.0000 & 4.0000 & 103.7401 & 279.3716 & 455.0031 & 0.0002\end{array}$

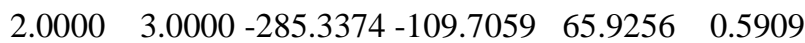




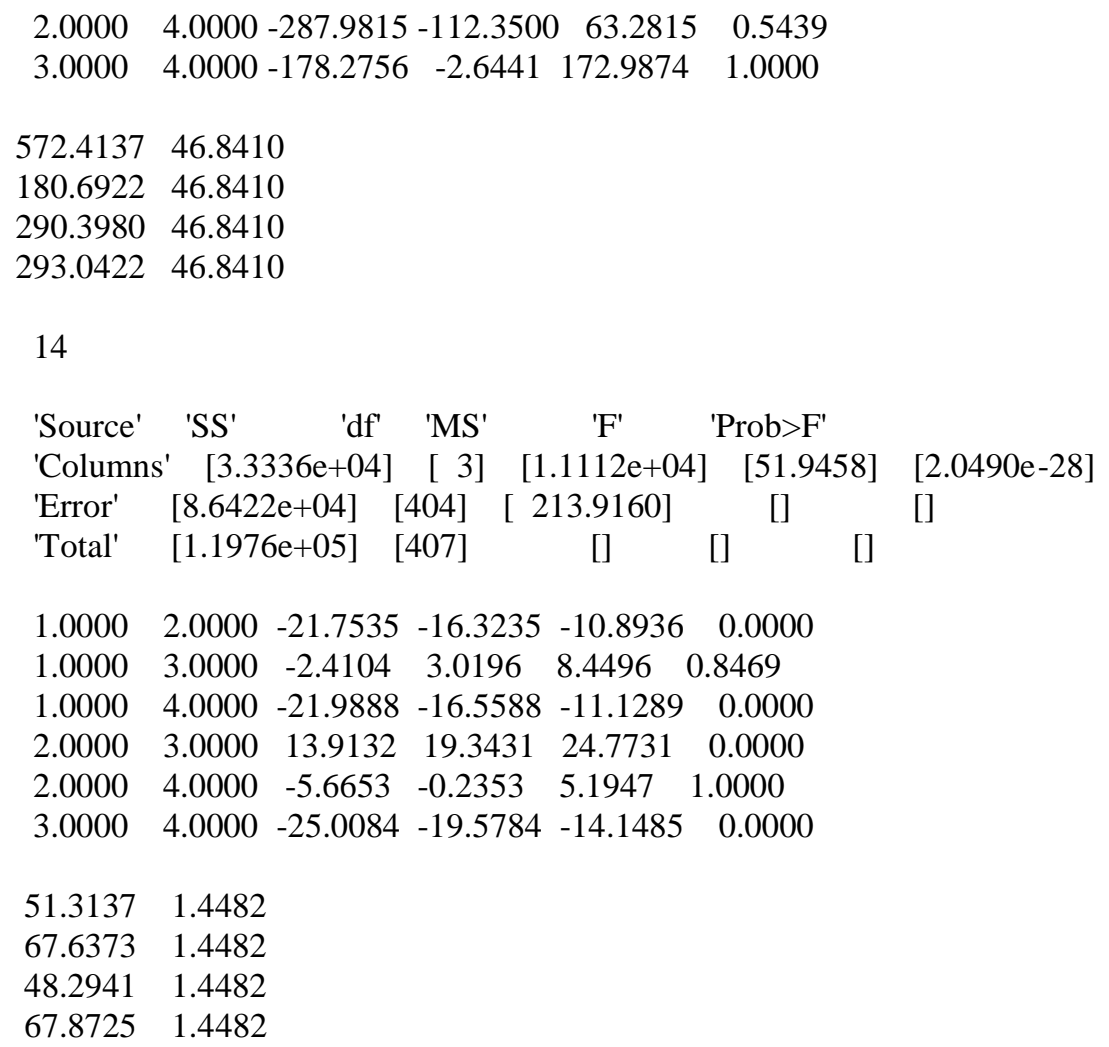

14

16

'Source' 'SS' 'df' 'MS' 'F' 'Prob>F' 'Columns' [1.8407e+03] [ 3] [613.5812] [4.7647] [0.0028] 


$\begin{array}{ll}15.2480 & 1.1236 \\ 19.7000 & 1.1236 \\ 20.8363 & 1.1236 \\ 19.4892 & 1.1236\end{array}$

17

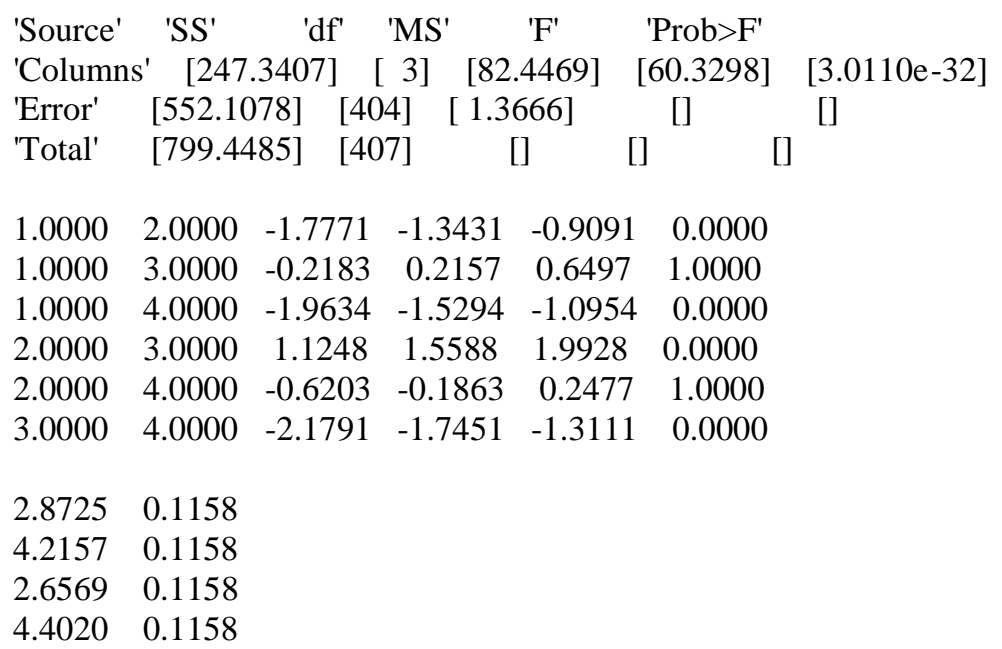

18

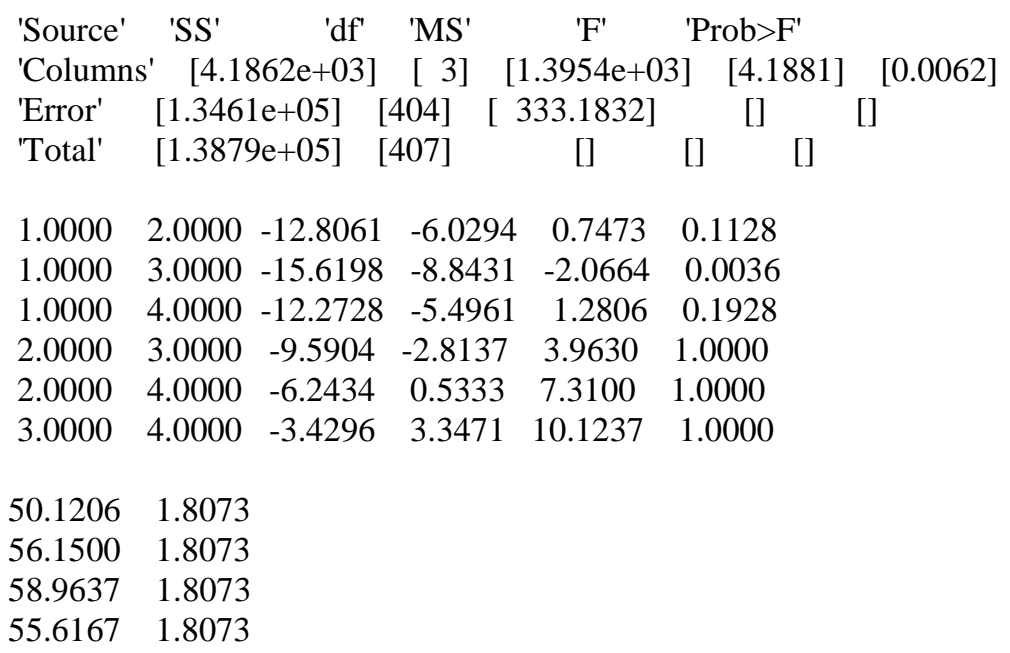

19

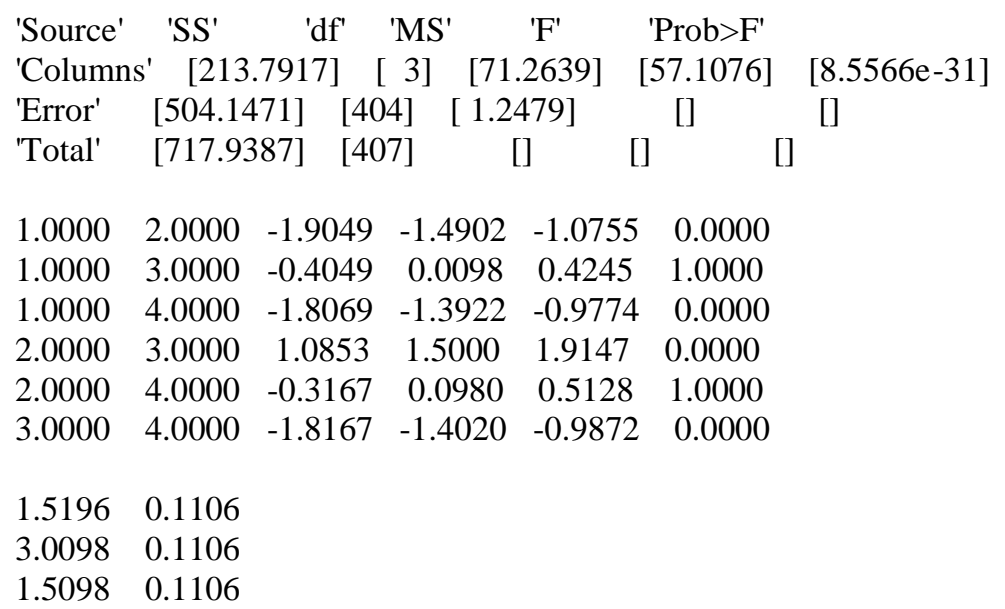


20

'Source' 'SS' 'df' 'MS' 'F' 'Prob>F'

'Columns' [ 93.5368] [ 3] [31.1789] [17.8099] [7.0970e-11]

$\begin{array}{llllll}\text { 'Error' } & {[707.2647]} & {[404]} & {[1.7507]} & {[]} & {[]}\end{array}$

'Total' [800.8015] [407] [] [] []

$\begin{array}{llllll}1.0000 & 2.0000 & -1.0795 & -0.5882 & -0.0970 & 0.0097\end{array}$

$\begin{array}{llllll}1.0000 & 3.0000 & 0.0088 & 0.5000 & 0.9912 & 0.0435\end{array}$

$\begin{array}{llllll}1.0000 & 4.0000 & -1.1775 & -0.6863 & -0.1951 & 0.0014\end{array}$

$\begin{array}{llllll}2.0000 & 3.0000 & 0.5970 & 1.0882 & 1.5795 & 0.0000\end{array}$

$\begin{array}{llllll}2.0000 & 4.0000 & -0.5893 & -0.0980 & 0.3932 & 1.0000\end{array}$

$\begin{array}{lllllll}3.0000 & 4.0000 & -1.6775 & -1.1863 & -0.6951 & 0.0000\end{array}$

$5.0784 \quad 0.1310$

$5.6667 \quad 0.1310$

$\begin{array}{ll}4.5784 & 0.1310\end{array}$

$5.7647 \quad 0.1310$

21

'Source' 'SS' 'df' 'MS' 'F' 'Prob>F'

'Columns' [196.2034] [ 3] [65.4011] [69.0171] [4.7243e-36]

'Error' [382.8333] [404] [0.9476]

'Total' [579.0368] [407]

[]

[] []

$\begin{array}{llllll}1.0000 & 2.0000 & -1.5477 & -1.1863 & -0.8249 & 0.0000\end{array}$

$\begin{array}{llllll}1.0000 & 3.0000 & -0.0379 & 0.3235 & 0.6849 & 0.1085\end{array}$

$\begin{array}{lllllll}1.0000 & 4.0000 & -1.5869 & -1.2255 & -0.8641 & 0.0000\end{array}$

$\begin{array}{lllllll}2.0000 & 3.0000 & 1.1484 & 1.5098 & 1.8712 & 0.0000\end{array}$

$\begin{array}{lllllll}2.0000 & 4.0000 & -0.4006 & -0.0392 & 0.3222 & 1.0000\end{array}$

$\begin{array}{lllllll}3.0000 & 4.0000 & -1.9104 & -1.5490 & -1.1876 & 0.0000\end{array}$

$3.0882 \quad 0.0964$

$4.2745 \quad 0.0964$

$2.7647 \quad 0.0964$

$4.3137 \quad 0.0964$

22

'Source' 'SS' 'df' 'MS' 'F'

'Columns' [ 32.8701] [ 3] [10.9567] [1.0507] [0.3700]

$\begin{array}{llllll}\text { 'Error' [4.2131e+03] [404] [10.4284] [] [] } & {[4]}\end{array}$

'Total' [4.2459e+03] [407] [] [] []

$\begin{array}{llllll}1.0000 & 2.0000 & -0.4342 & 0.7647 & 1.9636 & 0.5495\end{array}$

$\begin{array}{llllll}1.0000 & 3.0000 & -0.8264 & 0.3725 & 1.5715 & 1.0000\end{array}$

$\begin{array}{llllll}1.0000 & 4.0000 & -0.6205 & 0.5784 & 1.7773 & 1.0000\end{array}$

$\begin{array}{llllll}2.0000 & 3.0000 & -1.5911 & -0.3922 & 0.8067 & 1.0000\end{array}$

$\begin{array}{llllll}2.0000 & 4.0000 & -1.3852 & -0.1863 & 1.0126 & 1.0000\end{array}$

$\begin{array}{llllll}3.0000 & 4.0000 & -0.9930 & 0.2059 & 1.4048 & 1.0000\end{array}$

$4.6078 \quad 0.3197$

$3.8431 \quad 0.3197$

$4.2353 \quad 0.3197$

$4.0294 \quad 0.3197$

23 


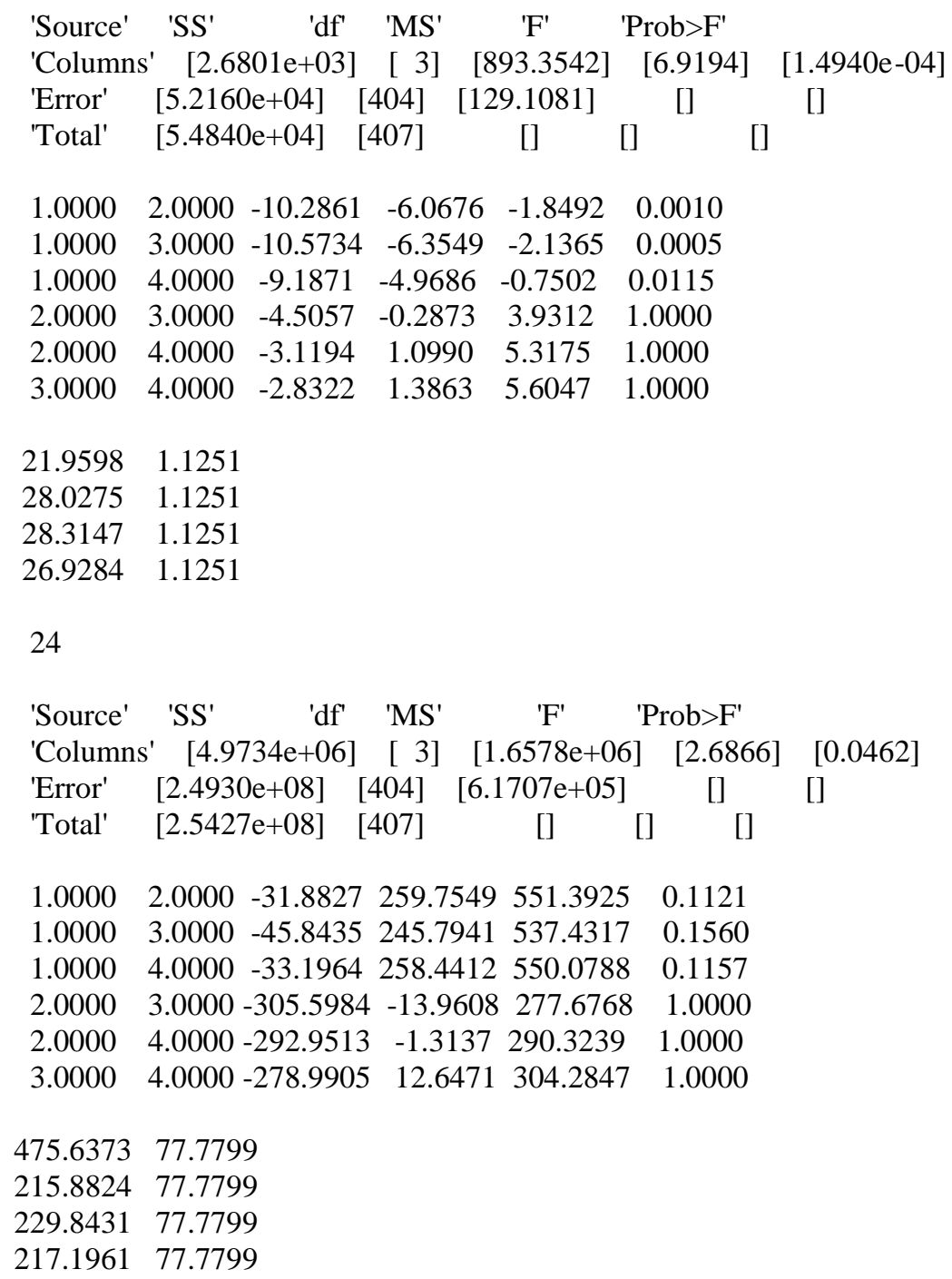




\begin{tabular}{|c|c|c|c|c|c|c|c|}
\hline \multicolumn{2}{|c|}{ 'Error' } & \multirow{2}{*}{\multicolumn{2}{|c|}{$[2.4903 e+05]$}} & [404] [ 6 & $516.4171]$ & [] & \multirow[t]{2}{*}{ [] } \\
\hline & & & & [407] & [] & [] & \\
\hline & 000 & 2.0000 & -27.0852 & -17.8676 & -8.6501 & 0.0000 & \\
\hline & 000 & 3.0000 & -17.0440 & -7.8265 & 1.3910 & 0.1495 & \\
\hline & 000 & 4.0000 & -24.1401 & -14.9225 & -5.7050 & 0.0001 & \\
\hline & 000 & 3.0000 & 0.8237 & 10.0412 & 19.2587 & 0.0245 & \\
\hline & 000 & 4.0000 & -6.2724 & 2.9451 & 12.1626 & 1.0000 & \\
\hline & 000 & 4.0000 & -16.3136 & -7.0961 & 2.1214 & 0.2513 & \\
\hline & 7059 & 2.4583 & & & & & \\
\hline & 5735 & 2.4583 & & & & & \\
\hline & 5324 & 2.4583 & & & & & \\
\hline & 5284 & 2.4583 & & & & & \\
\hline 27 & & & & & & & \\
\hline & urce' & 'SS' & 'df' 'MS & $S^{\prime} \quad ' F^{\prime}$ & Prob $>F^{\prime}$ & & \\
\hline & lumns & & [ 3] & 0] $[\mathrm{NaN}]$ & ] $[\mathrm{NaN}$ & & \\
\hline & ror' & {$[0]$} & {$[404] \quad[0]$} & ]$\quad[]$ & [] & & \\
\hline & tal' & {$[0]$} & [407] [] & [] & [] & & \\
\hline 1 & 2 & $0 \quad 0$ & $0 \mathrm{NaN}$ & & & & \\
\hline 1 & 3 & $\begin{array}{ll}0 & 0\end{array}$ & $0 \quad \mathrm{NaN}$ & & & & \\
\hline 1 & 4 & 0 & $0 \quad \mathrm{NaN}$ & & & & \\
\hline 2 & 3 & $0 \quad 0$ & $0 \quad \mathrm{NaN}$ & & & & \\
\hline 2 & 4 & $0 \quad 0$ & $0 \mathrm{NaN}$ & & & & \\
\hline 3 & 4 & $0 \quad 0$ & $0 \mathrm{NaN}$ & & & & \\
\hline 1 & 0 & & & & & & \\
\hline 1 & 0 & & & & & & \\
\hline 1 & 0 & & & & & & \\
\hline 1 & 0 & & & & & & \\
\hline
\end{tabular}

28

$\begin{array}{lccccccc}\text { 'Source' } & \text { 'SS' } & \text { 'df' } & \text { 'MS' } & \text { 'F' } & \text { 'Prob>F' } & \\ \text { 'Columns' } & {[1.0745 \mathrm{e}+03]} & {[3]} & {[358.1789]} & {[26.3636]} & {[1.3601 \mathrm{e}-15]} \\ \text { 'Error' } & {[5.4888 \mathrm{e}+03]} & {[404]} & {[13.5861]} & {[]} & {[]} \\ \text { 'Total' } & {[6.5633 \mathrm{e}+03]} & {[407]} & {[]} & {[]} & {[]} & \\ & & & & & & & \\ 1.0000 & 2.0000 & 2.7394 & 4.1078 & 5.4763 & 0.0000 & \\ 1.0000 & 3.0000 & 1.1610 & 2.5294 & 3.8978 & 0.0000 & \\ 1.0000 & 4.0000 & 2.4551 & 3.8235 & 5.1920 & 0.0000 & \\ 2.0000 & 3.0000 & -2.9469 & -1.5784 & -0.2100 & 0.0143 & \\ 2.0000 & 4.0000 & -1.6527 & -0.2843 & 1.0841 & 1.0000 & \\ 3.0000 & 4.0000 & -0.0743 & 1.2941 & 2.6626 & 0.0753 & \\ & & & & & & & \\ 8.1961 & 0.3650 & & & & & & \\ 4.0882 & 0.3650 & & & & & & \\ 5.6667 & 0.3650 & & & & & \end{array}$

29

'Source' 'SS' ' $\mathrm{df}^{\prime} \quad$ 'MS' 'F'

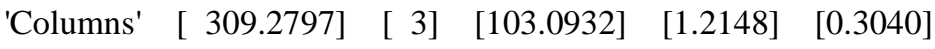

$\begin{array}{llllll}\text { 'Error' } & {[3.4286 \mathrm{e}+04]} & \text { [404] } & {[84.8674]} & \text { [ ] [ }\end{array}$

'Total' [3.4596e+04] [407] [] [] [ ] 


$\begin{array}{lrrrrr}1.0000 & 2.0000 & -1.0898 & 2.3304 & 5.7506 & 0.4295 \\ 1.0000 & 3.0000 & -1.6849 & 1.7353 & 5.1555 & 1.0000 \\ 1.0000 & 4.0000 & -1.6996 & 1.7206 & 5.1407 & 1.0000 \\ 2.0000 & 3.0000 & -4.0153 & -0.5951 & 2.8251 & 1.0000 \\ 2.0000 & 4.0000 & -4.0300 & -0.6098 & 2.8104 & 1.0000 \\ 3.0000 & 4.0000 & -3.4349 & -0.0147 & 3.4055 & 1.0000 \\ & & & & & \\ 7.3167 & 0.9122 & & & & \\ 4.9863 & 0.9122 & & & & \\ 5.5814 & 0.9122 & & & & \\ 5.5961 & 0.9122 & & & & \end{array}$

30

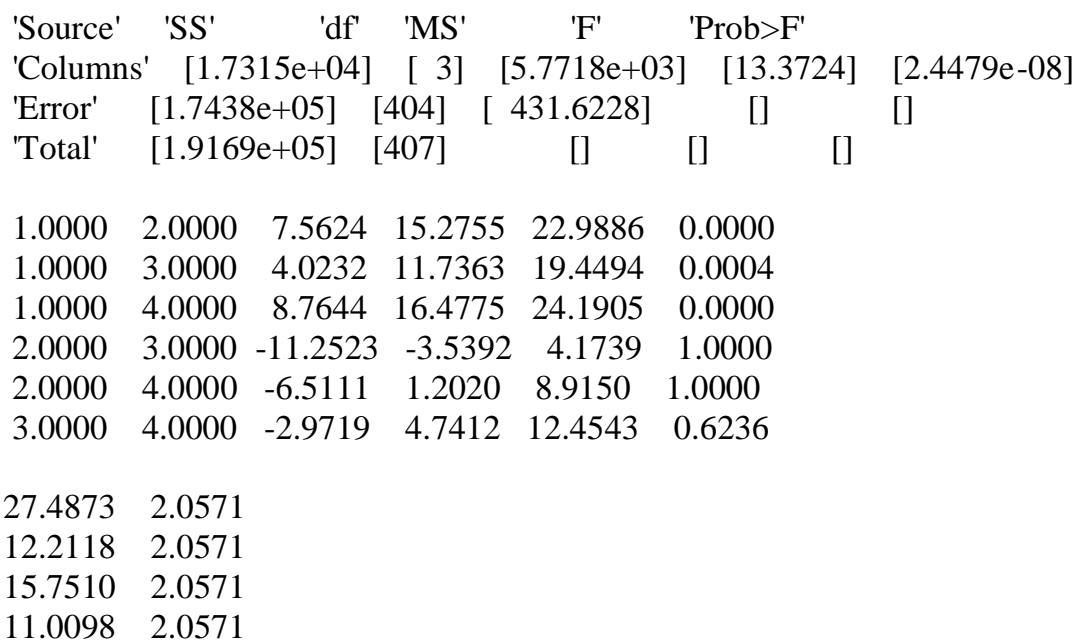

31

'Source' 'SS' 'df' 'MS' 'F' 'Prob $>F^{\prime}$

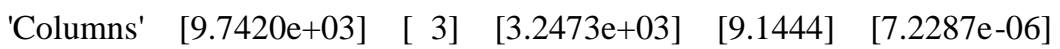

'Error' [1.4347e+05] [404] [ 355.1141] [] []

'Total' [1.5321e+05] [407] [] []

$\begin{array}{llllll}1.0000 & 2.0000 & 2.6401 & 9.6363 & 16.6324 & 0.0018\end{array}$

$\begin{array}{lllllll}1.0000 & 3.0000 & -2.0236 & 4.9725 & 11.9687 & 0.3613\end{array}$

$\begin{array}{llllll}1.0000 & 4.0000 & 5.9617 & 12.9578 & 19.9540 & 0.0000\end{array}$

$\begin{array}{llllll}2.0000 & 3.0000 & -11.6599 & -4.6637 & 2.3324 & 0.4675\end{array}$

$\begin{array}{llllll}2.0000 & 4.0000 & -3.6746 & 3.3216 & 10.3177 & 1.0000\end{array}$

$\begin{array}{llllll}3.0000 & 4.0000 & 0.9891 & 7.9853 & 14.9815 & 0.0158\end{array}$

$25.3686 \quad 1.8659$

$15.7324 \quad 1.8659$

$20.3961 \quad 1.8659$

$12.4108 \quad 1.8659$

32

'Source' 'SS' 'df' 'MS' 'F' 'Prob $>F^{\prime}$

'Columns' [7.1792e+03] [ 3] [2.3931e+03] [5.4077] [0.0012]

'Error' [1.7878e+05] [404] [ 442.5277] [] []

'Total' [1.8596e+05] [407] [] [] []

$\begin{array}{llllll}1.0000 & 2.0000 & 1.7695 & 9.5794 & 17.3893 & 0.0075\end{array}$

$\begin{array}{llllll}1.0000 & 3.0000 & 3.0391 & 10.8490 & 18.6589 & 0.0016\end{array}$

$\begin{array}{llllll}1.0000 & 4.0000 & -0.8187 & 6.9912 & 14.8011 & 0.1086\end{array}$ 


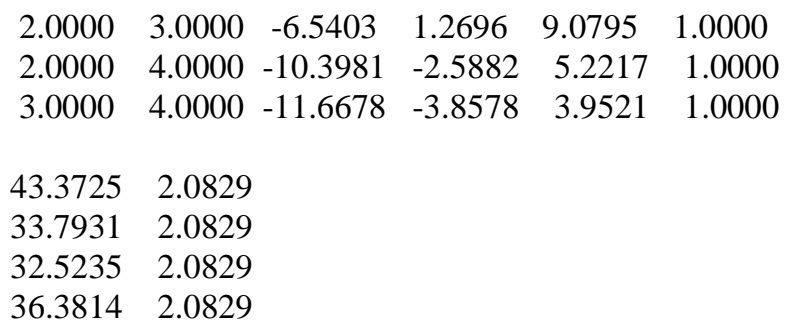

33

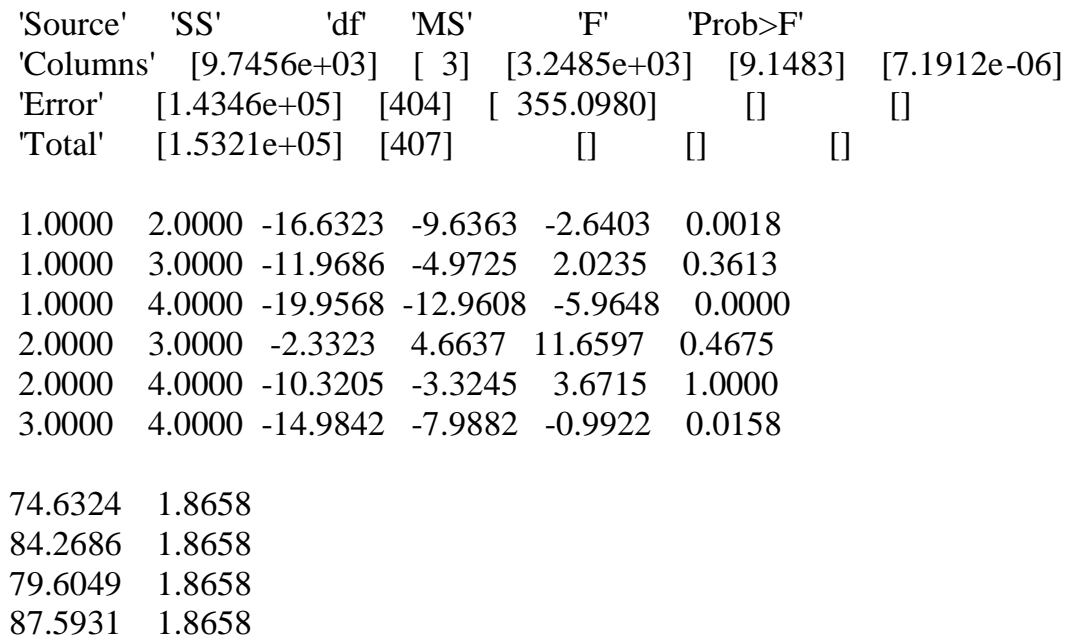




$\begin{array}{ll}77.0510 & 1.9015 \\ 86.8265 & 1.9015 \\ 81.9441 & 1.9015 \\ 91.1804 & 1.9015\end{array}$

36

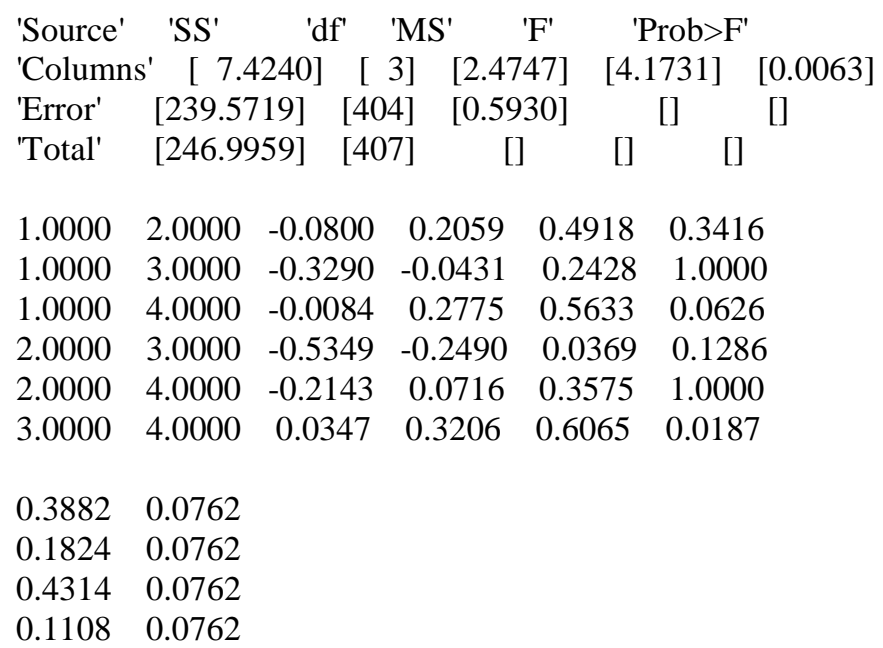

37

'Source' $\quad$ 'SS' $\quad$ 'df' $\quad$ 'MS' $\quad$ 'F' $\quad$ 'Prob $>F^{\prime}$

$\begin{array}{llllll} & \text { 'Columns' [3.6548e+03] [ 3] } & \text { [1.2183e+03] [11.8343] [1.9144e-07] }\end{array}$

$\begin{array}{llllllll}\text { 'Error' [4.1590e+04] [404] [ 102.9448] [] } & \text { [] }\end{array}$

$\begin{array}{llllll}\text { 'Total' [4.5245e+04] [407] [] [ ] } & \text { [ }\end{array}$

$\begin{array}{llllll}1.0000 & 2.0000 & 1.6047 & 5.3716 & 9.1384 & 0.0011\end{array}$

$\begin{array}{llllll}1.0000 & 3.0000 & -2.5914 & 1.1755 & 4.9423 & 1.0000\end{array}$

$\begin{array}{llllll}1.0000 & 4.0000 & 3.5645 & 7.3314 & 11.0982 & 0.0000\end{array}$

$\begin{array}{llllll}2.0000 & 3.0000 & -7.9629 & -4.1961 & -0.4292 & 0.0200\end{array}$

$\begin{array}{llllll}2.0000 & 4.0000 & -1.8070 & 1.9598 & 5.7267 & 1.0000\end{array}$

$\begin{array}{llllll}3.0000 & 4.0000 & 2.3890 & 6.1559 & 9.9227 & 0.0001\end{array}$

$12.2912 \quad 1.0046$

6.91961 .0046

$11.1157 \quad 1.0046$

$4.9598 \quad 1.0046$

38

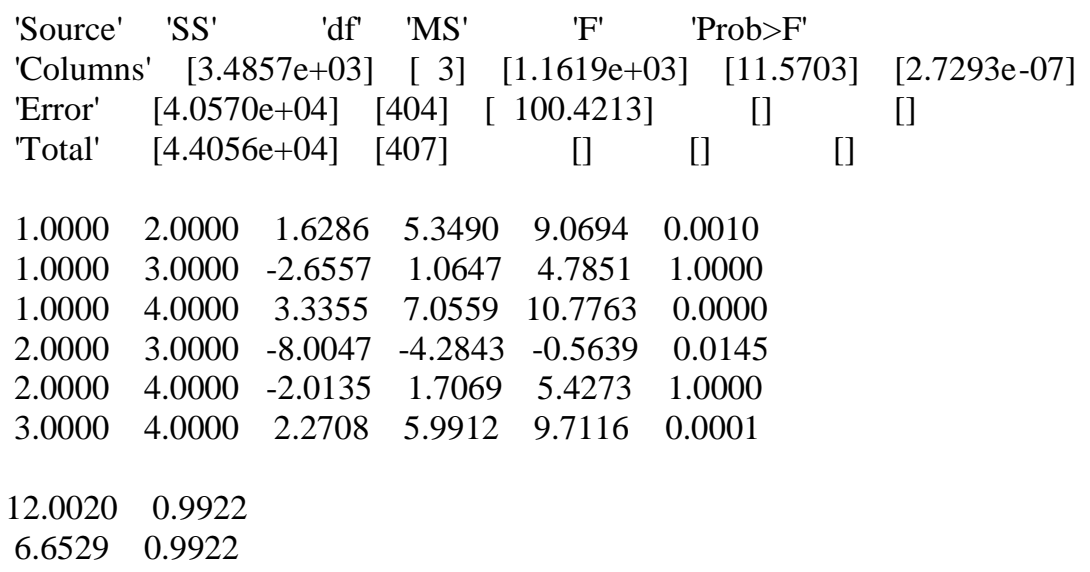


$10.9373 \quad 0.9922$

$4.9461 \quad 0.9922$

39

\begin{tabular}{|c|c|c|c|c|c|c|}
\hline 'Source' & 'SS' & 'df' & 'MS' & 'F' & 'Prob $>F^{\prime}$ & \\
\hline 'Columns & $s^{\prime} \quad[2.42$ & $63 e+03]$ & [ 3] [ & [808.7695] & [4.8011] & [0.0027] \\
\hline 'Error' & {$[6.8056$} & e+04] & [404] [1 & 168.4548] & [] & [] \\
\hline 'Total' & {$[7.0482$} & $e+04]$ & [407] & [] & [] & \\
\hline 1.0000 & 2.0000 & -0.4137 & 4.4049 & 9.2235 & 0.0948 & \\
\hline 1.0000 & 3.0000 & -1.0970 & 3.7216 & 8.5401 & 0.2474 & \\
\hline 1.0000 & 4.0000 & 1.9805 & 6.7990 & 11.6176 & 0.0013 & \\
\hline 2.0000 & 3.0000 & -5.5019 & -0.6833 & 34.1352 & 1.0000 & \\
\hline 2.0000 & 4.0000 & -2.4244 & 2.3941 & 7.2127 & 1.0000 & \\
\hline 3.0000 & 4.0000 & -1.7411 & 3.0775 & 7.8960 & 0.5470 & \\
\hline 10.6598 & 1.2851 & & & & & \\
\hline 6.2549 & 1.2851 & & & & & \\
\hline 6.9382 & 1.2851 & & & & & \\
\hline 3.8608 & 1.2851 & & & & & \\
\hline
\end{tabular}

40

\begin{tabular}{|c|c|c|c|c|c|}
\hline 'Source' & 'SS' & 'MS' & 'F' & \multicolumn{2}{|c|}{ 'Prob $>F^{\prime}$} \\
\hline 'Columns & $s^{\prime} \quad[2.7165 e+04]$ & ] [ 3] & {$[9.0551 \mathrm{e}+($} & \multicolumn{2}{|c|}{ 3] [11.8390] } \\
\hline 'Error' & {$[3.0900 \mathrm{e}+05]$} & [404] [ ] & 764.8483 & \multirow{2}{*}{\multicolumn{2}{|c|}{ [] [] }} \\
\hline 'Total' & {$[3.3616 \mathrm{e}+05]$} & [407] & [] & & \\
\hline 1.0000 & $2.0000 \quad-25.3851$ & $1-15.1176$ & $6-4.8502$ & \multicolumn{2}{|l|}{0.0007} \\
\hline 1.0000 & $3.0000-16.2136$ & $\begin{array}{ll}6 & -5.9461\end{array}$ & 14.3214 & \multicolumn{2}{|l|}{0.7528} \\
\hline 1.0000 & $4.0000-31.4459$ & $9-21.1784$ & $4-10.911$ & \multicolumn{2}{|l|}{0.0000} \\
\hline 2.0000 & $3.0000 \quad-1.0959$ & 9.1716 & 19.4390 & \multicolumn{2}{|l|}{0.1100} \\
\hline 2.0000 & $4.0000-16.3283$ & $3-6.0608$ & $8 \quad 4.2067$ & \multicolumn{2}{|l|}{0.7101} \\
\hline 3.0000 & $4.0000-25.4998$ & $8-15.2324$ & $4-4.9649$ & \multicolumn{2}{|l|}{0.0006} \\
\hline 65.0588 & 2.7383 & & & & \\
\hline 80.1765 & 2.7383 & & & & \\
\hline 71.0049 & 2.7383 & & & & \\
\hline 86.2373 & 2.7383 & & & & \\
\hline \multicolumn{6}{|l|}{41} \\
\hline 'Source' & 'SS' & 'MS' & 'F' & \\
\hline 'Columns & $s^{\prime} \quad[$ 159.6320] & {$[3] \quad[5$} & 53.2107] & \multicolumn{2}{|c|}{$\begin{array}{l}\text { 'Prob>F' } \\
{[1.5595] \quad[0.1987]}\end{array}$} \\
\hline 'Error' & {$[1.3785 \mathrm{e}+04]$} & [404] [32 & $34.1202]$ & \multicolumn{2}{|l|}{ [] } \\
\hline 'Total' & {$[1.3944 \mathrm{e}+04]$} & [407] & [] & [] & \\
\hline 1.0000 & $2.0000 \quad-1.2265$ & 0.9422 & 3.1108 & \multicolumn{2}{|l|}{1.0000} \\
\hline 1.0000 & $3.0000 \quad-0.6725$ & 1.4961 & 3.6647 & \multicolumn{2}{|l|}{0.4087} \\
\hline 1.0000 & $\begin{array}{ll}4.0000 & -2.1049\end{array}$ & 0.0637 & 2.2323 & \multicolumn{2}{|l|}{1.0000} \\
\hline 2.0000 & $3.0000 \quad-1.6147$ & 0.5539 & 2.7225 & \multicolumn{2}{|l|}{1.0000} \\
\hline 2.0000 & $4.0000 \quad-3.0470$ & -0.8784 & 1.2902 & \multicolumn{2}{|l|}{1.0000} \\
\hline 3.0000 & $4.0000 \quad-3.6010$ & -1.4324 & 0.7363 & \multicolumn{2}{|l|}{0.4840} \\
\hline 47.3382 & 0.5784 & & & & \\
\hline 46.3961 & 0.5784 & & & & \\
\hline 45.8422 & 0.5784 & & & & \\
\hline 47.2745 & 0.5784 & & & & \\
\hline
\end{tabular}




$\begin{array}{lccccccc}\text { 'Source' } & \text { 'SS' } & \text { 'df' } & \text { 'MS' } & \text { 'F' } & \text { 'Prob>F' } & \\ \text { 'Columns' } & {[148.5865]} & {[3]} & {[49.5288]} & {[1.2858]} & {[0.2788]} \\ \text { 'Error' } & {[1.5562 \mathrm{e}+04]} & {[404]} & {[38.5189]} & {[]} & {[]} \\ \text { 'Total' } & {[1.5710 \mathrm{e}+04]} & {[407]} & {[]} & {[]} & {[]} & \\ & & & & & & & \\ 1.0000 & 2.0000 & -3.6130 & -1.3088 & 0.9953 & 0.7971 & \\ 1.0000 & 3.0000 & -3.9071 & -1.6029 & 0.7012 & 0.3951 & \\ 1.0000 & 4.0000 & -3.2512 & -0.9471 & 1.3571 & 1.0000 & \\ 2.0000 & 3.0000 & -2.5983 & -0.2941 & 2.0100 & 1.0000 & \\ 2.0000 & 4.0000 & -1.9424 & 0.3618 & 2.6659 & 1.0000 & \\ 3.0000 & 4.0000 & -1.6483 & 0.6559 & 2.9600 & 1.0000 & \\ & & & & & & & \\ 48.7794 & 0.6145 & & & & & & \\ 50.0882 & 0.6145 & & & & & & \\ 50.3824 & 0.6145 & & & & & & \end{array}$

43

'Source' 'SS' 'df' 'MS' 'F' $\quad$ 'Prob>F'

'Columns' [ 46.7434] [ 3] [15.5811] [1.3817] [0.2479]

'Error' [4.5559e+03] [404] [11.2771] [] []

'Total' [4.6027e+03] [407] [] [ ]

$\begin{array}{llllll}1.0000 & 2.0000 & -0.8781 & 0.3686 & 1.6154 & 1.0000\end{array}$

$\begin{array}{llllll}1.0000 & 3.0000 & -1.1438 & 0.1029 & 1.3497 & 1.0000\end{array}$

$\begin{array}{llllll}1.0000 & 4.0000 & -0.3722 & 0.8745 & 2.1212 & 0.3819\end{array}$

$\begin{array}{llllll}2.0000 & 3.0000 & -1.5124 & -0.2657 & 0.9811 & 1.0000\end{array}$

$\begin{array}{llllll}2.0000 & 4.0000 & -0.7409 & 0.5059 & 1.7526 & 1.0000\end{array}$

$\begin{array}{llllll}3.0000 & 4.0000 & -0.4752 & 0.7716 & 2.0183 & 0.6097\end{array}$

$3.8814 \quad 0.3325$

$3.5127 \quad 0.3325$

$3.7784 \quad 0.3325$

$3.0069 \quad 0.3325$

44

'Source' 'SS' ' $\mathrm{df}^{\prime} \quad$ 'MS' 'F' 'Prob>F'

'Columns' [2.4905e+03] [ 3] [830.1573] [8.3336] [2.1755e-05]

'Error' [4.0245e+04] [404] [ 99.6163] [] []

'Total' [4.2735e+04] [407] [] []

$\begin{array}{llllll}1.0000 & 2.0000 & -9.3731 & -5.6676 & -1.9622 & 0.0004\end{array}$

$\begin{array}{llllll}1.0000 & 3.0000 & -9.7760 & -6.0706 & -2.3651 & 0.0001\end{array}$

$\begin{array}{llllll}1.0000 & 4.0000 & -6.2662 & -2.5608 & 1.1447 & 0.4059\end{array}$

$\begin{array}{llllll}2.0000 & 3.0000 & -4.1084 & -0.4029 & 3.3025 & 1.0000\end{array}$

$\begin{array}{llllll}2.0000 & 4.0000 & -0.5986 & 3.1069 & 6.8123 & 0.1606\end{array}$

$\begin{array}{llllll}3.0000 & 4.0000 & -0.1956 & 3.5098 & 7.2153 & 0.0745\end{array}$

$7.2873 \quad 0.9882$

$12.9549 \quad 0.9882$

$13.3578 \quad 0.9882$

$9.8480 \quad 0.9882$

45

'Source' 'SS' $\quad$ 'df' $\quad$ 'MS' $\quad$ 'F' $\quad$ 'Prob $>F^{\prime}$ 


\begin{tabular}{|c|c|c|c|c|c|c|}
\hline 'Columns' & \multicolumn{2}{|c|}{$\quad[2.5202 \mathrm{e}+03]$} & \multicolumn{2}{|c|}{ [ [3] [840.0622] } & [8.3797] & {$[2.0432 \mathrm{e}$} \\
\hline 'Error' & {$[4.0501$} & $e+04]$ & [404] [1 & $00.2498]$ & [] & [] \\
\hline 'Total' & [4.3021 & $e+04]$ & [407] & [] & [] & ] \\
\hline 1.0000 & 2.0000 & 1.9965 & 5.7137 & 9.4309 & 0.0003 & \\
\hline 1.0000 & 3.0000 & 2.3720 & 6.0892 & 9.8064 & 0.0001 & \\
\hline 1.0000 & 4.0000 & -1.1584 & 2.5588 & 6.2760 & 0.4124 & \\
\hline 2.0000 & 3.0000 & -3.3417 & 0.3755 & 4.0927 & 1.0000 & \\
\hline 2.0000 & 4.0000 & -6.8721 & -3.1549 & 0.5623 & 0.1498 & \\
\hline 3.0000 & 4.0000 & -7.2476 & -3.5304 & 0.1868 & 0.0731 & \\
\hline 92.7127 & 0.9914 & & & & & \\
\hline 86.9990 & 0.9914 & & & & & \\
\hline 86.6235 & 0.9914 & & & & & \\
\hline 90.1539 & 0.9914 & & & & & \\
\hline 46 & & & & & & \\
\hline 'Source' & 'SS' & 'df' & 'MS' & 'F' & 'Prob $>F^{\prime}$ & \\
\hline 'Columns' & ' $\quad[2.28$ & $99 e+03]$ & [ 3] & $763.3158]$ & [2.7818] & [0.0407] \\
\hline 'Error' & {$[1.1086$} & $e+05]$ & {$[404] \quad[2$} & $74.3974]$ & [] & {[]} \\
\hline 'Total' & {$[1.1315$} & $e+05]$ & [407] & [] & {$\left[\begin{array}{ll}] & {[}\end{array}\right]$} & \\
\hline 1.0000 & 2.0000 & -0.7656 & 5.3843 & 11.5342 & 0.1246 & \\
\hline 1.0000 & 3.0000 & -0.3724 & 5.7775 & 11.9273 & 0.0789 & \\
\hline 1.0000 & 4.0000 & -0.9646 & 5.1853 & 11.3352 & 0.1556 & \\
\hline 2.0000 & 3.0000 & -5.7567 & 0.3931 & 6.5430 & 1.0000 & \\
\hline 2.0000 & 4.0000 & -6.3489 & -0.1990 & 5.9509 & 1.0000 & \\
\hline 3.0000 & 4.0000 & -6.7420 & -0.5922 & 5.5577 & 1.0000 & \\
\hline 22.2010 & 1.6402 & & & & & \\
\hline 16.8167 & 1.6402 & & & & & \\
\hline 16.4235 & 1.6402 & & & & & \\
\hline 17.0157 & 1.6402 & & & & & \\
\hline
\end{tabular}

47

'Source' 'SS' 'df' 'MS' 'F' 'Prob>F'

$\begin{array}{llllll} & \text { 'Columns' [2.1603e+03] [ 3] [720.0922] [0.7753] } & \text { [0.5083] }\end{array}$

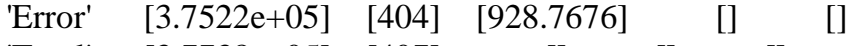

$\begin{array}{llllll}\text { 'Total' [3.7738e+05] [407] [ ] [ ] } & {[]}\end{array}$

$\begin{array}{llllll}1.0000 & 2.0000 & -15.5898 & -4.2755 & 7.0389 & 1.0000\end{array}$

$\begin{array}{llllll}1.0000 & 3.0000 & -14.2957 & -2.9814 & 8.3330 & 1.0000\end{array}$

$\begin{array}{llllll}1.0000 & 4.0000 & -9.8065 & 1.5078 & 12.8222 & 1.0000\end{array}$

$\begin{array}{llllll}2.0000 & 3.0000 & -10.0202 & 1.2941 & 12.6085 & 1.0000\end{array}$

$\begin{array}{llllll}2.0000 & 4.0000 & -5.5310 & 5.7833 & 17.0977 & 1.0000\end{array}$

$\begin{array}{llllll}3.0000 & 4.0000 & -6.8251 & 4.4892 & 15.8036 & 1.0000\end{array}$

$59.5716 \quad 3.0175$

63.84713 .0175

$62.5529 \quad 3.0175$

$58.0637 \quad 3.0175$

48

'Source' 'SS' 'df' 'MS' 'F' 'Prob>F'

$\begin{array}{llllll}\text { 'Columns' [ 971.1589] } & \text { [ 3] } & \text { [323.7196] [1.9957] } & \text { [0.1140] }\end{array}$

$\begin{array}{llllll}\text { 'Error' } & {[6.5532 \mathrm{e}+04]} & {[404]} & {[162.2086]} & {[]} & {[]}\end{array}$

'Total' [6.6503e+04] [407] [] [] [] 


$\begin{array}{llllll}1.0000 & 2.0000 & -8.7872 & -4.0588 & 0.6696 & 0.1403 \\ 1.0000 & 3.0000 & -6.6549 & -1.9265 & 2.8019 & 1.0000 \\ 1.0000 & 4.0000 & -8.0284 & -3.3000 & 1.4284 & 0.3899 \\ 2.0000 & 3.0000 & -2.5960 & 2.1324 & 6.8607 & 1.0000 \\ 2.0000 & 4.0000 & -3.9696 & 0.7588 & 5.4872 & 1.0000 \\ 3.0000 & 4.0000 & -6.1019 & -1.3735 & 3.3549 & 1.0000 \\ & & & & & \\ 13.0373 & 1.2611 & & & & \\ 17.0961 & 1.2611 & & & & \\ 14.9637 & 1.2611 & & & & \\ 16.3373 & 1.2611 & & & & \end{array}$

49

'Source' 'SS' 'df' 'MS' 'F' 'Prob>F'

'Columns' [ 339.2491] [ 3] [113.0830] [5.7791] [7.0787e-04]

'Error' [7.9053e+03] [404] [ 19.5675] [] []

'Total' [8.2445e+03] [407] [] []

$\begin{array}{llllll}1.0000 & 2.0000 & 0.4175 & 2.0598 & 3.7021 & 0.0058\end{array}$

$\begin{array}{llllll}1.0000 & 3.0000 & -0.6129 & 1.0294 & 2.6717 & 0.5838\end{array}$

$\begin{array}{llllll}1.0000 & 4.0000 & 0.6548 & 2.2971 & 3.9393 & 0.0014\end{array}$

$\begin{array}{llllll}2.0000 & 3.0000 & -2.6727 & -1.0304 & 0.6119 & 0.5819\end{array}$

$\begin{array}{llllll}2.0000 & 4.0000 & -1.4050 & 0.2373 & 1.8795 & 1.0000\end{array}$

$\begin{array}{llllll}3.0000 & 4.0000 & -0.3746 & 1.2676 & 2.9099 & 0.2481\end{array}$

$3.8186 \quad 0.4380$

$\begin{array}{ll}1.7588 & 0.4380\end{array}$

$2.7892 \quad 0.4380$

$1.5216 \quad 0.4380$

50

'Source' 'SS' 'df' 'MS' 'F' 'Prob>F'

'Columns' [ 597.2689] [ 3] [199.0896] [2.8348] [0.0380]

$\begin{array}{llllll}\text { 'Error' [2.8373e+04] [404] [ [70.2309] [] } & \text { [] }\end{array}$

'Total' [2.8971e+04] [407] [] [] []

$\begin{array}{llllll}1.0000 & 2.0000 & -4.7750 & -1.6637 & 1.4476 & 0.9422\end{array}$

$\begin{array}{llllll}1.0000 & 3.0000 & -1.6407 & 1.4706 & 4.5819 & 1.0000\end{array}$

$\begin{array}{llllll}1.0000 & 4.0000 & -4.2936 & -1.1824 & 1.9289 & 1.0000\end{array}$

$\begin{array}{llllll}2.0000 & 3.0000 & 0.0230 & 3.1343 & 6.2456 & 0.0472\end{array}$

$\begin{array}{llllll}2.0000 & 4.0000 & -2.6299 & 0.4814 & 3.5927 & 1.0000\end{array}$

$\begin{array}{llllll}3.0000 & 4.0000 & -5.7642 & -2.6529 & 0.4583 & 0.1458\end{array}$

$\begin{array}{ll}11.5235 & 0.8298\end{array}$

$13.1873 \quad 0.8298$

$10.0529 \quad 0.8298$

12.70590 .8298

51

'Source' 'SS' 'df' 'MS' 'F' $\quad$ 'Prob>F'

'Columns' [ 337.1848] [ 3] [112.3949] [3.3569] [0.0189]

'Error' [1.3527e+04] [404] [ 33.4815] [] []

'Total' [1.3864e+04] [407] [] [] []

$\begin{array}{llllll}1.0000 & 2.0000 & -2.1335 & 0.0147 & 2.1629 & 1.0000\end{array}$

$\begin{array}{lllllll}1.0000 & 3.0000 & -2.7453 & -0.5971 & 1.5512 & 1.0000\end{array}$ 


$\begin{array}{rrrrrr}1.0000 & 4.0000 & -0.3217 & 1.8265 & 3.9747 & 0.1483 \\ 2.0000 & 3.0000 & -2.7600 & -0.6118 & 1.5365 & 1.0000 \\ 2.0000 & 4.0000 & -0.3365 & 1.8118 & 3.9600 & 0.1554 \\ 3.0000 & 4.0000 & 0.2753 & 2.4235 & 4.5717 & 0.0177 \\ & & & & & \\ 3.2118 & 0.5729 & & & & \\ 3.1971 & 0.5729 & & & & \\ 3.8088 & 0.5729 & & & & \\ 1.3853 & 0.5729 & & & & \end{array}$

52

$\begin{array}{lccccccc}\text { 'Source' } & \text { 'SS' } & \text { 'df' } & \text { 'MS' } & \text { 'F' } & \text { 'Prob>F' } & \\ \text { 'Columns' } & {[335.5158]} & {[3]} & {[111.8386]} & {[1.4697]} & {[0.2222]} \\ \text { 'Error' } & {[3.0742 \mathrm{e}+04]} & {[404]} & {[76.0940]} & {[]} & {[]} \\ \text { 'Total' } & {[3.1077 \mathrm{e}+04]} & {[407]} & {[]} & {[]} & {[]} & \\ & & & & & & & \\ 1.0000 & 2.0000 & -4.6788 & -1.4402 & 1.7984 & 1.0000 & \\ 1.0000 & 3.0000 & -5.4248 & -2.1863 & 1.0523 & 0.4454 & \\ 1.0000 & 4.0000 & -5.4925 & -2.2539 & 0.9846 & 0.3944 & \\ 2.0000 & 3.0000 & -3.9846 & -0.7461 & 2.4925 & 1.0000 & \\ 2.0000 & 4.0000 & -4.0523 & -0.8137 & 2.4248 & 1.0000 & \\ 3.0000 & 4.0000 & -3.3062 & -0.0676 & 3.1709 & 1.0000 & \\ & & & & & & & \\ 11.1784 & 0.8637 & & & & & & \end{array}$

53

'Source' 'SS' 'df' 'MS' 'F' 'Prob>F'

'Columns' [ 123.9025] [ 3] [41.3008] [0.4641] [0.7075]

'Error' [3.5952e+04] [404] [88.9901] [] []

'Total' [3.6076e+04] [407] [] [] []

$\begin{array}{llllll}1.0000 & 2.0000 & -4.2179 & -0.7157 & 2.7866 & 1.0000\end{array}$

$\begin{array}{llllll}1.0000 & 3.0000 & -4.8640 & -1.3618 & 2.1405 & 1.0000\end{array}$

$\begin{array}{llllll}1.0000 & 4.0000 & -4.8130 & -1.3108 & 2.1915 & 1.0000\end{array}$

$\begin{array}{llllll}2.0000 & 3.0000 & -4.1483 & -0.6461 & 2.8562 & 1.0000\end{array}$

$\begin{array}{llllll}2.0000 & 4.0000 & -4.0973 & -0.5951 & 2.9072 & 1.0000\end{array}$

$\begin{array}{llllll}3.0000 & 4.0000 & -3.4513 & 0.0510 & 3.5532 & 1.0000\end{array}$

$10.7627 \quad 0.9341$

$11.4784 \quad 0.9341$

$12.1245 \quad 0.9341$

$12.0735 \quad 0.9341$

54

'Source' 'SS' 'df' 'MS' 'F' 'Prob>F'

'Columns' [ 362.2468] [ 3] [120.7489] [4.3395] [0.0050]

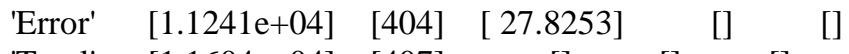

'Total' [1.1604e+04] [407] [] [] []

$\begin{array}{llllll}1.0000 & 2.0000 & -0.3496 & 1.6088 & 3.5672 & 0.1799\end{array}$

$\begin{array}{llllll}1.0000 & 3.0000 & -1.8152 & 0.1431 & 2.1015 & 1.0000\end{array}$

$\begin{array}{llllll}1.0000 & 4.0000 & 0.2446 & 2.2029 & 4.1613 & 0.0182\end{array}$

$\begin{array}{lllllll}2.0000 & 3.0000 & -3.4241 & -1.4657 & 0.4927 & 0.2874\end{array}$

$\begin{array}{llllll}2.0000 & 4.0000 & -1.3643 & 0.5941 & 2.5525 & 1.0000\end{array}$ 


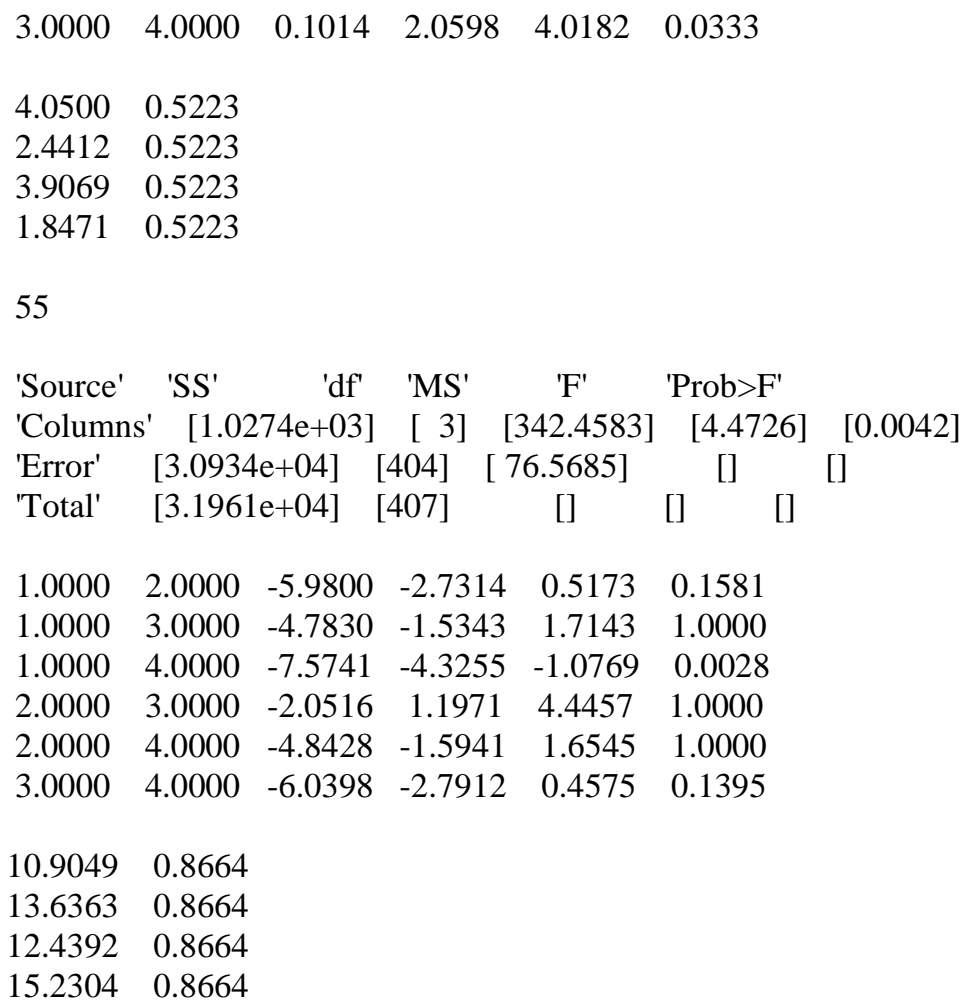

55

'Source' 'SS' 'df' 'MS' 'F' 'Prob>F'

$\begin{array}{lllllll} & \text { 'Columns' [1.0274e+03] [ 3] [342.4583] [4.4726] [0.0042] }\end{array}$

$\begin{array}{llllll}\text { 'Error' [3.0934e+04] [404] [ 76.5685] [] } & \text { [ ] }\end{array}$

'Total' [3.1961e+04] [407] [] [] []

$\begin{array}{llllll}1.0000 & 2.0000 & -5.9800 & -2.7314 & 0.5173 & 0.1581\end{array}$

$\begin{array}{llllll}1.0000 & 3.0000 & -4.7830 & -1.5343 & 1.7143 & 1.0000\end{array}$

$\begin{array}{lllllll}1.0000 & 4.0000 & -7.5741 & -4.3255 & -1.0769 & 0.0028\end{array}$

$\begin{array}{llllll}2.0000 & 3.0000 & -2.0516 & 1.1971 & 4.4457 & 1.0000\end{array}$

$\begin{array}{llllll}2.0000 & 4.0000 & -4.8428 & -1.5941 & 1.6545 & 1.0000\end{array}$

$\begin{array}{llllll}3.0000 & 4.0000 & -6.0398 & -2.7912 & 0.4575 & 0.1395\end{array}$

$10.9049 \quad 0.8664$

$13.6363 \quad 0.8664$

$12.4392 \quad 0.8664$

$15.2304 \quad 0.8664$

56

'Source' 'SS' 'df' 'MS' 'F' 'Prob>F'

'Columns' [ 378.4441] [ 3] [126.1480] [5.1131] [0.0018]

'Error' [9.9673e+03] [404] [ 24.6715] [] []

'Total' [1.0346e+04] [407] [] [] []

$\begin{array}{llllll}1.0000 & 2.0000 & -0.2293 & 1.6147 & 3.4588 & 0.1245\end{array}$

$\begin{array}{llllll}1.0000 & 3.0000 & -0.5735 & 1.2706 & 3.1146 & 0.4108\end{array}$

$\begin{array}{llllll}1.0000 & 4.0000 & 0.8550 & 2.6990 & 4.5431 & 0.0007\end{array}$

$\begin{array}{llllll}2.0000 & 3.0000 & -2.1882 & -0.3441 & 1.4999 & 1.0000\end{array}$

$\begin{array}{llllll}2.0000 & 4.0000 & -0.7597 & 1.0843 & 2.9284 & 0.7187\end{array}$

$\begin{array}{llllll}3.0000 & 4.0000 & -0.4156 & 1.4284 & 3.2725 & 0.2439\end{array}$

$4.1755 \quad 0.4918$

$2.5608 \quad 0.4918$

$2.9049 \quad 0.4918$

$1.4765 \quad 0.4918$

57

'Columns' [ 280.4071] [ 3] [93.4690] [1.3521] [0.2571]

$\begin{array}{ccccccc}\text { 'Error' } & {[2.7928 \mathrm{e}+04]} & {[404]} & {[69.1298]} & {[]} & & {[]} \\ \text { 'Total' } & {[2.8209 \mathrm{e}+04]} & {[407]} & {[]} & {[]} & {[]}\end{array}$

$\begin{array}{llllll}1.0000 & 2.0000 & -2.1201 & 0.9667 & 4.0535 & 1.0000\end{array}$

$\begin{array}{llllll}1.0000 & 3.0000 & -1.6290 & 1.4578 & 4.5446 & 1.0000\end{array}$

$\begin{array}{llllll}1.0000 & 4.0000 & -3.7613 & -0.6745 & 2.4123 & 1.0000\end{array}$

$\begin{array}{llllll}2.0000 & 3.0000 & -2.5956 & 0.4912 & 3.5780 & 1.0000\end{array}$

$\begin{array}{lllllll}2.0000 & 4.0000 & -4.7280 & -1.6412 & 1.4456 & 0.9565\end{array}$

$\begin{array}{llllll}3.0000 & 4.0000 & -5.2192 & -2.1324 & 0.9544 & 0.4066\end{array}$

$11.7637 \quad 0.8233$ 
$10.7971 \quad 0.8233$

$10.3059 \quad 0.8233$

$12.4382 \quad 0.8233$

58

'Source' 'SS' ' $\mathrm{df}^{\prime} \quad$ 'MS' 'F' $\quad$ 'Prob>F'

'Columns' [ 455.2134] [ 3] [151.7378] [5.7296] [7.5739e-04]

$\begin{array}{lllcccc}\text { 'Error' } & {[1.0699 \mathrm{e}+04]} & {[404]} & {[26.4833]} & \text { [] } & \text { [] } \\ \text { 'Total' } & {[1.1154 \mathrm{e}+04]} & {[407]} & {[]} & {[]} & {[]}\end{array}$

$\begin{array}{llllll}1.0000 & 2.0000 & -0.2537 & 1.6569 & 3.5674 & 0.1320\end{array}$

$\begin{array}{llllll}1.0000 & 3.0000 & -1.1066 & 0.8039 & 2.7145 & 1.0000\end{array}$

$\begin{array}{lllllll}1.0000 & 4.0000 & 0.9394 & 2.8500 & 4.7606 & 0.0005\end{array}$

$\begin{array}{llllll}2.0000 & 3.0000 & -2.7635 & -0.8529 & 1.0576 & 1.0000\end{array}$

$\begin{array}{llllll}2.0000 & 4.0000 & -0.7174 & 1.1931 & 3.1037 & 0.5913\end{array}$

$\begin{array}{llllll}3.0000 & 4.0000 & 0.1355 & 2.0461 & 3.9566 & 0.0285\end{array}$

$4.2961 \quad 0.5095$

$2.6392 \quad 0.5095$

3.49220 .5095

$1.4461 \quad 0.5095$

59

'Source' 'SS' ' 'df' 'MS' 'F' $\quad$ 'Prob>F'

'Columns' [ 376.1065] [ 3] [125.3688] [1.9041] [0.1283]

'Error' [2.6600e+04] [404] [65.8418] [] []

'Total' [2.6976e+04] [407] $\left[\begin{array}{llll}\text { [ } & \text { [] } & \text { [] }\end{array}\right.$

$\begin{array}{llllll}1.0000 & 2.0000 & -0.3096 & 2.7029 & 5.7154 & 0.1070\end{array}$

$\begin{array}{llllll}1.0000 & 3.0000 & -1.5919 & 1.4206 & 4.4331 & 1.0000\end{array}$

$\begin{array}{llllll}1.0000 & 4.0000 & -1.8419 & 1.1706 & 4.1831 & 1.0000\end{array}$

$\begin{array}{llllll}2.0000 & 3.0000 & -4.2949 & -1.2824 & 1.7301 & 1.0000\end{array}$

$\begin{array}{llllll}2.0000 & 4.0000 & -4.5449 & -1.5324 & 1.4801 & 1.0000\end{array}$

$\begin{array}{llllll}3.0000 & 4.0000 & -3.2625 & -0.2500 & 2.7625 & 1.0000\end{array}$

$\begin{array}{ll}11.2735 & 0.8034\end{array}$

$8.5706 \quad 0.8034$

$9.8529 \quad 0.8034$

$10.1029 \quad 0.8034$ 